\title{
KOSZUL DUALITY FOR IWASAWA ALGEBRAS MODULO $p$
}

\author{
CLAUS SORENSEN
}

\begin{abstract}
In this article we establish a version of Koszul duality for filtered rings arising from $p$-adic Lie groups. Our precise setup is the following. We let $G$ be a uniform pro- $p$ group and consider its completed group algebra $\Omega=k \llbracket G \rrbracket$ with coefficients in a finite field $k$ of characteristic $p$. It is known that $\Omega$ carries a natural filtration and $\operatorname{gr} \Omega=S(\mathfrak{g})$ where $\mathfrak{g}$ is the (abelian) Lie algebra of $G$ over $k$. One of our main results in this paper is that the Koszul dual $\operatorname{gr} \Omega^{!}=\wedge \mathfrak{g}^{\vee}$ can be promoted to an $A_{\infty}$-algebra in such a way that the derived category of pseudocompact $\Omega$-modules $D(\Omega)$ becomes equivalent to the derived category of strictly unital $A_{\infty}$-modules $D_{\infty}\left(\bigwedge \mathfrak{g}^{\vee}\right)$. In the case where $G$ is an abelian group we prove that the $A_{\infty}$-structure is trivial and deduce an equivalence between $D(\Omega)$ and the derived category of differential graded modules over $\bigwedge \mathfrak{g}^{\vee}$ which generalizes a result of Schneider for $\mathbb{Z}_{p}$.
\end{abstract}

\section{Contents}

1. Introduction

2. Notation

3. Pontryagin duality for profinite groups

4. Keller's Morita theorem for DGA's

5 . Schneider's equivalence for $p$-adic Lie groups

6. The Yoneda algebra of $\Omega$

7. Review of Lazard's theory of $p$-valued groups

8. Mod $p$ cohomology of equi- $p$-valued groups

9. $A_{\infty}$-algebras and their minimal models

10. $A_{\infty}$-modules and the derived category

11. Putting the pieces together

12. Open ends and unanswered questions

\section{INTRODUCTION}

Classical Koszul duality is a derived equivalence between modules over the symmetric algebra $S(V)$ and modules over the exterior algebra $\wedge V^{\vee}$. Here $V$ is a finite-dimensional vector space over a field $k$, and $V^{\vee}=\operatorname{Hom}_{k}(V, k)$ is its dual space. More precisely one considers the category of finitely generated graded left modules over $S(V)$ and its bounded derived category $D^{b}(S(V))$. Similarly for the

Received by the editors March 18, 2019, and, in revised form, January 7, 2020.

2010 Mathematics Subject Classification. Primary 20C08, 22E35, 13D09. 
exterior algebra. Then Koszul duality gives an equivalence of triangulated categories

$$
D^{b}(S(V)) \stackrel{\sim}{\longrightarrow} D^{b}\left(\bigwedge V^{\vee}\right) .
$$

This is BGG78, Thm. 3]. The equivalence of course derives its name from the Koszul resolution

$$
\cdots \longrightarrow S(V) \otimes \bigwedge^{2} V \longrightarrow S(V) \otimes \bigwedge^{1} V \longrightarrow S(V) \longrightarrow k \longrightarrow 0 .
$$

Building on work of Priddy, Löfwall, Backelin, and others, this was generalized to more general rings in BGS96. They define a Koszul algebra to be a graded $k$-algebra $A=k \oplus A_{1} \oplus A_{2} \oplus \cdots$ for which $k$ admits a (graded) projective resolution $P_{\bullet} \rightarrow k$ such that each $P_{i}$ is generated by its degree $i$ component $\left(P_{i}=A P_{i, i}\right)$. The Koszul dual algebra $A^{!}$is defined by viewing $A$ as a quadratic algebra and then dualizing the relations; cf. BGS96, Def. 2.8.1]. Alternatively one can identify it with the (opposite) Yoneda algebra $A^{!}=\operatorname{Ext}_{A}^{*}(k, k)^{\text {op }}$ of self-extensions of $k$; cf. [BGS96, Thm. 2.10.1]. Assuming $A$ is locally finite-dimensional, that is, $\operatorname{dim}_{k}\left(A_{i}\right)<$ $\infty$ for all $i$, the Koszul dual $A^{!}$is again Koszul and canonically $A^{! !} \stackrel{\sim}{\longrightarrow} A$. In the classical case $A=S(V)$ has Koszul dual $A^{!}=\bigwedge V^{\vee} \simeq \operatorname{Ext}_{S(V)}^{*}(k, k)^{\mathrm{op}}$. Still under the assumption that $A$ is locally finite-dimensional, [BGS96, Thm. 2.12.1] establishes an equivalence

$$
D^{\downarrow}(A) \stackrel{\sim}{\longrightarrow} D^{\uparrow}\left(A^{!}\right)
$$

where $D^{\downarrow}$ and $D^{\uparrow}$ are variants of the derived categories. For instance $D^{\downarrow}(A)$ is obtained by inverting quasi-isomorphisms in the category $C^{\downarrow}(A)$ of complexes of graded $A$-modules $\cdots \rightarrow M^{i} \rightarrow M^{i+1} \rightarrow \cdots$ satisfying $M_{j}^{i}=0$ if $i \ll 0$ or $i+j \gg 0$ (no other finiteness assumptions on the $M^{i}$ ). Assuming $A$ itself is finite-dimensional (hence $A_{i}=0$ for $i \gg 0$ ) and that $A^{!}$is Noetherian, [BGS96, Thm. 2.12.6] gives an equivalence between bounded derived categories

$$
D^{b}(A) \stackrel{\sim}{\longrightarrow} D^{b}\left(A^{!}\right) .
$$

Here, as in the classical case, we are deriving the categories of finitely generated graded modules. Some of the finiteness assumptions in BGS96 have since been weakened in MOS09.

There have been several attempts to extend Koszul duality to filtered algebras, notably the works Pos93, Flo06] of Fløystad and Positselski, which share some important features with the main results of this paper. Given a Koszul algebra $A$ as above, Positselski considers filtered rings $U$ with $\operatorname{gr}(U) \simeq A$ and shows that this

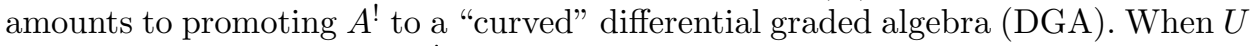
is furthermore augmented $A^{!}$is an actual DGA. (This is our main case of interest, so we will not recall here what it means to be curved.) Fløystad took this further and constructed a pair of adjoint functors between homotopy categories $K(U) \rightleftarrows$ $K\left(A^{!}, d\right)$. He showed they descend to an equivalence $\tilde{D}(U) \stackrel{\sim}{\longrightarrow} \tilde{D}\left(A^{!}, d\right)$, but here $\tilde{D}$ is not the derived category; it is rather squeezed in between the homotopy category and the derived category. We should emphasize that the filtered rings $U$ considered in Pos93 are of a completely different nature than the Iwasawa algebras we will consider in this paper. Positselski requires $U$ to be a non-homogeneous quadratic algebra $U=T(V) /(P)$ for some vector space $V$ and ideal of relations $(P)$ in the tensor algebra generated by a subspace $P \subset k \oplus V \oplus V^{\otimes 2}$ with $P \cap(k \oplus V)=0$. 
He associates the quadratic algebra $A=T(V) /(Q)$ where $Q \subset V^{\otimes 2}$ is the image of $P$ under projection and decrees that the natural map $A \rightarrow \operatorname{gr} U$ is an isomorphism.

Analogously we will consider completed group rings $\Omega=\Omega(G)$ modulo $p$ of certain compact $p$-adic Lie groups $G$ for which $\operatorname{gr} \Omega \simeq S(\mathfrak{g})$, where $\mathfrak{g}=\operatorname{Lie}(G)$ is the Lie algebra, and promote the Koszul dual algebra gr $\Omega^{!} \simeq \wedge \mathfrak{g}^{\vee}$ to a so-called $A_{\infty^{-}}$ algebra in such a way that $D(\Omega) \stackrel{\sim}{\longrightarrow} D_{\infty}\left(\bigwedge \mathfrak{g}^{\vee}\right)$. The notation and terminology will be explained in more detail below.

Let us state our main results precisely. Let $G$ be a $p$-adic Lie group which is torsionfre 1 and pro- $p$. Let $\Omega=k \llbracket G \rrbracket$ be its completed group ring over a finite field $k$ of characteristic $p$, and consider the derived category $D(\Omega)$ of the category of pseudocompact left $\Omega$-modules.

Theorem 1.1. The (opposite) Yoneda algebra $\Omega^{!}:=\operatorname{Ext}_{\Omega}^{*}(k, k)^{\text {op }}$ has an $A_{\infty}$ algebra structure, defined uniquely up to $A_{\infty}$-isomorphism, for which there is an equivalence of triangulated categories

$$
D(\Omega) \stackrel{\sim}{\longrightarrow} D_{\infty}\left(\Omega^{!}\right)
$$

where the target is the derived category of strictly unital left $A_{\infty}$-modules over $\Omega$ !

When $G$ is a uniform pro- $p$ group (see the discussion after Corollary 8.3) one can compute $\Omega$ ! using Lazard's calculation of the mod $p$ cohomology ring of an equi- $p$-valued group and deduce the next result.

Theorem 1.2. Let $G$ be a uniform pro-p group with Lie algebra $\mathfrak{g}$ over $k$ (which is necessarily abelian). Then $\bigwedge \mathfrak{g}^{\vee}$ has an $A_{\infty}$-algebra structure, defined uniquely up to $A_{\infty}$-isomorphism, for which there is an equivalence of triangulated categories

$$
D(\Omega) \stackrel{\sim}{\longrightarrow} D_{\infty}\left(\bigwedge \mathfrak{g}^{\vee}\right) .
$$

Furthermore, when $G$ is abelian the $A_{\infty}$-algebra structure on $\bigwedge \mathfrak{g}^{\vee}$ is trivial (meaning all higher multiplication maps $\mu_{n}$ vanish for $\left.n>2\right)$ and the target is the derived category of differential graded modules.

For example this theorem applies to the congruence subgroups $G=\operatorname{ker}\left(\mathcal{G}\left(\mathbb{Z}_{p}\right) \rightarrow\right.$ $\mathcal{G}\left(\mathbb{Z} / p^{m} \mathbb{Z}\right)$ ) of any finite type smooth affine group scheme $\mathcal{G}_{/ \mathbb{Z}_{p}}-$ at least for $m \gg 0$. Let us also point out that any $p$-adic Lie group contains a uniform open subgroup; cf. Laz65.

The case $G=\mathbb{Z}_{p}$ of Theorem 1.2 is a result of Schneider. In [Sch15, p. 460] it is shown that there is an equivalence $D\left(\mathbb{Z}_{p}\right) \stackrel{\sim}{\longrightarrow} D(k[\varepsilon])$ where $k[\varepsilon]=k \oplus$ $k \varepsilon$ is the algebra of dual numbers $\left(\varepsilon^{2}=0\right)$ thought of as a differential graded algebra concentrated in degrees 0 and 1 with zero differential. This is a special case of Theorem 1.2 by observing that $k[\varepsilon] \simeq \bigwedge k$, and a computation in Hochschild cohomology shows $\bigwedge k$ is intrinsically formal - it has no non-trivial minimal $A_{\infty^{-}}$ structures whatsoever; cf. Remark 11.4 for more details.

Schneider's paper Sch15 in fact plays a pivotal role in the proof of our two theorems, which we will now outline. The equivalence in Theorem 1.1 factors as a composition (after taking opposites)

$$
D(\Omega)^{\mathrm{op}} \stackrel{(1)}{=} D(G) \stackrel{(2)}{=} D\left(\mathcal{H}^{\bullet}\right) \stackrel{(3)}{=} D_{\infty}\left(h^{*}\left(\mathcal{H}^{\bullet}\right)\right) \stackrel{(4)}{=} D_{\infty}\left(\operatorname{Ext}_{\Omega}^{*}(k, k)\right) .
$$

\footnotetext{
${ }^{1}$ Positselski has kindly informed us that Theorem 1.1 can be extended to any pro- $p$ group if one replaces $D(\Omega)^{\text {op }}$ by the so-called coderived category of smooth $G$-modules by applying the results from Pos11, Sec. 6.4].
} 
Here we use $=$ instead of $\stackrel{\sim}{\longrightarrow}$ for clarity. We explain each step (1)-(4) separately.

(1) (Section 3) The first step is just Pontryagin duality between the category $\operatorname{Mod}(\Omega)$ of pseudocompact $\Omega$-modules and the smooth $k[G]$-modules $\operatorname{Rep}_{k}^{\infty}(G)$. Taking continuous $k$-linear duals defines mutually quasi-inverse contravariant functors which are exact and therefore induce an anti-equivalence on the level of derived categories.

(2) (Section 5) This is the main result of [Sch15] in the special case $G=I$. We discuss this case in some detail in the main body of the text but claim no novelty here (except for the exposition). The result is based on a theorem of Keller which says that any algebraic triangulated category $\mathcal{D}$ with (coproducts and) a compact generator $C$ is equivalent to $D\left(\mathcal{H}_{C}^{\bullet}\right)$ for some naturally defined differential graded algebra $\mathcal{H}_{C}^{\bullet}$; see section 4 . Schneider shows that Keller's theorem applies to $\mathcal{D}=D(G)$ for a torsionfree pro- $p$ group $G$ and $C=k$ is the trivial representation. The crucial step is to verify that $k$ is a generator of $D(G)$; cf. Proposition 5.4 below. For that we need $G$ to be torsionfree to guarantee it has finite cohomological $p$-dimension. The DGA is here simply $\mathcal{H}^{\bullet}=\operatorname{End}_{G}^{\bullet}\left(I^{\bullet}\right)^{\text {op }}$ for a choice of injective resolution $k \rightarrow I^{\bullet}$. Alternatively $\mathcal{H}^{\bullet}=\operatorname{End}_{\Omega}^{\bullet}\left(P^{\bullet}\right)$ for a choice of projective resolution $P^{\bullet} \rightarrow k$, by Pontryagin duality.

(3) (Sections 9 and 10) By a general result of Kadeishvili from the 1980s the cohomology algebra $h^{*}\left(A^{\bullet}\right)$ of any DGA $A^{\bullet}$ (even of any $A_{\infty}$-algebra) has an $A_{\infty}$-structure $\left(\mu_{n}\right)_{n \geq 1}$ with $\mu_{1}=0$ ("minimality") and $\mu_{2}$ induced by multiplication on $A^{\bullet}$, for which there is a quasi-isomorphism $f: h^{*}\left(A^{\bullet}\right) \rightarrow$ $A^{\bullet}$. We will discuss this in detail in section 9. Here we will just note that each $\mu_{n}$ is a higher multiplication map $A^{\bullet \otimes n} \rightarrow A^{\bullet}$ of degree $2-n$ and the sequence $\left(\mu_{n}\right)_{n \geq 1}$ satisfies generalized associativity laws known as the Stasheff identities. One can restrict $A_{\infty}$-modules along $f$, and this gives rise to an equivalence $D\left(A^{\bullet}\right) \stackrel{\sim}{\longrightarrow} D_{\infty}\left(h^{*}\left(A^{\bullet}\right)\right)$ as discussed in section 10 , The latter is due to Lefèvre-Hasegawa. Applying this to $\mathcal{H}^{\bullet}$ yields (3).

(4) (Section 6) This is merely the computation $h^{*}\left(\mathcal{H}^{\bullet}\right)=h^{*}\left(\operatorname{End}_{\Omega}^{\bullet}\left(P^{\bullet}\right)\right)=$ $\operatorname{Ext}_{\Omega}^{*}(k, k)$.

The first half of Theorem 1.2 is a corollary of Theorem 1.1 by calculating $\operatorname{Ext}_{\Omega}^{*}(k, k)$ for uniform pro- $p$ groups (equivalently $G$, which admits a $p$-valuation $\omega$ such that $(G, \omega)$ is equi- $p$-valued; see section 7). Namely,

$$
\Omega^{!}=\operatorname{Ext}_{\Omega}^{*}(k, k)^{\mathrm{op}}=\operatorname{Ext}_{G}^{*}(k, k)=H^{*}(G, k)=\bigwedge \mathfrak{g}^{\vee},
$$

where the last step is due to Lazard; cf. Corollary 8.3 below. The second half (where $G$ is abelian) is proved in section 11.2. The basic idea is to think of $\Omega$ as a completed symmetric algebra $\Omega=\widehat{S(V)}$ and take $P^{\bullet} \rightarrow k$ to be the completion of the Koszul

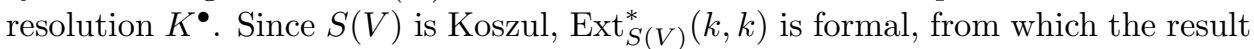
follows after observing that there is a quasi-isomorphism $\operatorname{End}_{S(V)}^{\bullet}\left(K^{\bullet}\right) \rightarrow \mathcal{H}^{\bullet}$.

In fact Theorem 1.1 has a natural extension to arbitrary $p$-adic Lie groups $G$ (not necessarily compact). As in Sch15 fix a torsionfree pro- $p$ subgroup $I \subset G$. Schneider shows that $\operatorname{ind}_{I}^{G}(1)$ is a compact generator of $D(G)$ and deduces an equivalence with $D\left(\mathcal{H}^{\bullet}\right)$ from Keller's theorem. Here the Hecke DGA is defined as $\mathcal{H}^{\bullet}=\operatorname{End}_{G}^{\bullet}\left(I^{\bullet}\right)^{\text {op }}$ for a choice of injective resolution $\operatorname{ind}_{I}^{G}(1) \longrightarrow I^{\bullet}$. The Iwasawa algebra is defined to be $\Omega=k[G] \otimes_{k[I]} k \llbracket I \rrbracket$, and Pontryagin duality extends to 
an equivalence $D(\Omega)^{\mathrm{op}}=D(G)$ as explained in [Koh17, Thm. 1.5]. Altogether the $\bmod p$ "derived Hecke algebra" (cf. Ven19])

$$
\Omega^{!}:=\operatorname{Ext}_{G}^{*}\left(\operatorname{ind}_{I}^{G}(1), \operatorname{ind}_{I}^{G}(1)\right) \simeq H^{*}\left(I, \operatorname{ind}_{I}^{G}(1)\right)
$$

admits an $A_{\infty}$-structure such that $D(\Omega) \stackrel{\sim}{\longrightarrow} D_{\infty}\left(\Omega^{!}\right)$. We have chosen not to emphasize this stronger result since the computation of $H^{*}\left(I, \operatorname{ind}_{I}^{G}(1)\right)$ appears to be quite delicate in general. The recent paper [OS18] deals with the case $\mathrm{SL}_{2}(F)$ for arbitrary $F / \mathbb{Q}_{p}$, which is already quite involved. See OS18, Thm. 3.23] for sample calculations.

We should point out that Theorems 1.1 and 1.2 were known to some experts in the field, although they never appeared explicitly in print. Also, the $A_{\infty}$-structures we work with are only uniquely defined up to a non-canonical $A_{\infty}$-isomorphism; they depend on several choices. See the end of section 9 for instance. This severely limits the usage of our results beyond the case of abelian groups $G$. However, we feel that Theorems 1.1 and 1.2 are of some interest in their own right, at least from a philosophical standpoint.

In order to make this article more accessible to mathematicians from different disciplines, we have included lots of details and background on the Keller-Schneider equivalence, Lazard's theory of $p$-valued groups and their cohomology, $A_{\infty}$-algebras and modules, and other material relevant for the paper.

\section{Notation}

Throughout $p$ denotes a fixed odd prime. Similar arguments most likely go through for $p=2$ with minor modifications, but we have not checked this in detail. One issue is the notion of a uniform pro- $p$ group $G$ in the case $p=2$ where one should require that $G / \overline{G^{4}}$ be abelian.

If $\mathcal{A}$ is an abelian category its opposite category $\mathcal{A}^{\text {op }}$ is again an abelian category; kernels in $\mathcal{A}$ correspond to cokernels in $\mathcal{A}^{\text {op }}$ and vice versa (similarly for images and coimages); cf. [Sta18, Lem. 12.5.2]. If $\mathcal{C}$ is any category, when we view $X \in \mathrm{Ob}(\mathcal{C})$ as an object of $\mathcal{C}^{\text {op }}$ we will write $X^{\text {op }}$ to avoid confusion; similarly for morphisms.

A triangulated category is an additive category $\mathcal{D}$ with a translation functor $T: \mathcal{D} \rightarrow \mathcal{D}$ (an auto-equivalence) and a class of distinguished triangles $X \rightarrow Y \rightarrow$ $Z \rightarrow T X$ satisfying four axioms TR1-4; cf. [Sta18, Def. 13.3.2]. The opposite category $\mathcal{D}^{\mathrm{op}}$ is again a triangulated category. Its translation functor is the inverse $T^{-1}$, and the triangle $X^{\mathrm{op}} \rightarrow Y^{\mathrm{op}} \rightarrow Z^{\mathrm{op}} \rightarrow T^{-1} X^{\mathrm{op}}$ is declared to be distinguished precisely when $T^{-1} X \rightarrow Z \rightarrow Y \rightarrow X$ is distinguished in $\mathcal{D}$; cf. [Nee01, Rem. 1.1.5].

If $\mathcal{A}$ is an abelian category $C(\mathcal{A})$ denotes the category of complexes $\cdots \rightarrow X^{i} \rightarrow$ $X^{i+1} \rightarrow \cdots$. We let $K(\mathcal{A})$ be the homotopy category and let $D(\mathcal{A})$ be the (unbounded) derived category; both are triangulated categories in the usual fashion; cf. Har66, Ch. I]. Taking opposites is compatible with derived categories, to the effect that there is a natural equivalence of triangulated categories $D\left(\mathcal{A}^{\mathrm{op}}\right) \stackrel{\sim}{\longrightarrow} D(\mathcal{A})^{\mathrm{op}}$.

\section{Pontryagin DUality For PROFinite Groups}

In this section $G$ denotes an arbitrary profinite group and $k$ is any field. We let $\Omega=\Omega(G)=k \llbracket G \rrbracket$ be the Iwasawa $k$-algebra of $G$. We will usually suppress $G$ from the notation and just write $\Omega$. It has a natural augmentation map $\Omega \stackrel{a}{\longrightarrow} k$ and carries the inverse limit topology. We always endow $k$ with the discrete topology. Thus $\Omega=\lim _{\longleftarrow} k[G / N]$ (where $N \subset G$ runs over the open normal subgroups) is a 
pseudocompact ring, meaning it is a complete Hausdorff topological ring which has a fundamental system of open neighborhoods of zero consisting of two-sided ideals $I_{N}$ such that $\Omega / I_{N}$ is Artinian.

In this paper we will mostly be interested in the category $\operatorname{Mod}(\Omega)$ of pseudocompact left $\Omega$-modules (and continuous homomorphisms). Its objects can be defined as pseudocompact $k$-vector spaces $M$ (that is, inverse limits of finite-dimensional $k$-vector spaces) with a $k$-linear jointly continuous action $G \times M \longrightarrow M$. One can immediately verify that the $k[G]$-module structure of $M$ then extends uniquely to a continuous map $\Omega \times M \longrightarrow M$ and that $M$ admits a neighborhood basis at zero consisting of $\Omega$-submodules.

It is well-known that $\operatorname{Mod}(\Omega)$ is an abelian category which admits arbitrary products, and the formation of filtered (inverse) limits is exact; cf. Gab62, p. 392]. Moreover $\operatorname{Mod}(\Omega)$ has enough projectives; cf. Bru66, Lem. 1.6].

Dually we have the abelian category $\operatorname{Rep}_{k}^{\infty}(G)$ of smooth $G$-representations on $k$-vector spaces $V$ (and $k[G]$-linear maps). In other words the action $G \times V \longrightarrow V$ is continuous for the discrete topology on $V$. The Pontryagin dual of $V$ is defined as $V^{\vee}=\operatorname{Hom}_{k}(V, k)$ equipped with the topology of pointwise convergence (i.e., induced from the product topology on $\left.k^{V}\right)$. With the contragredient $G$-action $V^{\vee}$ thus becomes an object of $\operatorname{Mod}(\Omega)$. Conversely, starting from a pseudocompact $\Omega$ module $M$ we define its dual to be $M^{\vee}=\operatorname{Hom}_{k}^{\text {cts }}(M, k)$ with the discrete topology. It is well-known that this sets up a duality between the two categories. See Sch95, ST02, Eme10, and the more recent [Koh17. We summarize this below.

Theorem 3.1. The Pontryagin duality functors $(\cdot)^{\vee}$ are exact and mutually quasiinverse. Thus

$$
(\cdot)^{\vee}: \operatorname{Mod}(\Omega)^{o p} \longrightarrow \operatorname{Rep}_{k}^{\infty}(G)
$$

is an equivalence of categories. Furthermore $\Omega^{\vee}=\mathcal{C}^{\infty}(G, k)$.

Proof. Apart from exactness this is essentially [Koh17, Thm. 1.5] in the case of a compact group.

In particular we infer that $\operatorname{Rep}_{k}^{\infty}(G)$ admits arbitrary coproducts (direct sums), that filtered colimits are exact, and it has enough injectives. In fact $\operatorname{Rep}_{k}^{\infty}(G)$ is a Grothendieck category since it has a generator $Y=\bigoplus_{N} \operatorname{ind}_{N}^{G}(1)$. Indeed, Frobenius reciprocity $\operatorname{Hom}_{G}(Y, V)=\prod_{N} V^{N}$ shows that the functor $\operatorname{Hom}_{G}(Y,-)$ is faithful.

Because $(\cdot)^{\vee}$ is exact it preserves quasi-isomorphisms and induces an equivalence on the level of derived categories. Throughout the paper we let $D(G)=$ $D\left(\operatorname{Rep}_{k}^{\infty}(G)\right)$ and $D(\Omega)=D(\operatorname{Mod}(\Omega))$. Similarly for the category of complexes $C(G)$ and the homotopy category $K(G)$. The same for $\Omega$.

Corollary 3.2. The functors $(\cdot)^{\vee}$ define an equivalence of triangulated categories $D(\Omega)^{\mathrm{op}} \stackrel{\sim}{\longrightarrow} D(G)$.

Here the opposite triangulated category $D(\Omega)^{\text {op }}$ is equivalent to $D\left(\operatorname{Mod}(\Omega)^{\mathrm{op}}\right)$ as explained in the notation section, section 2

\section{Keller's Morita theorem for DGA's}

If $\mathcal{A}$ is an abelian category with coproducts which admits a compact (i.e., $\operatorname{Hom}_{\mathcal{A}}(P,-)$ commutes with coproducts) projective generator $P$, then a theorem of Morita states that $\operatorname{Hom}_{\mathcal{A}}(P,-)$ gives an equivalence $\mathcal{A} \longrightarrow \operatorname{Mod}\left(\operatorname{End}_{\mathcal{A}}(P)^{\text {op }}\right)$ onto the category of left modules over the algebra $\operatorname{End}_{\mathcal{A}}(P)^{\mathrm{op}}$. 
In Kel94 Keller proved an analogue of Morita's theorem for differential graded algebras (from now on referred to as DGA's). Here we will follow [Kel07] in exposing his result. First recall that a DGA over a field $k$ is a $\mathbb{Z}$-graded associative $k$-algebra $\mathcal{H}^{\bullet}=\bigoplus_{i \in \mathbb{Z}} \mathcal{H}^{i}$ with a degree one $k$-linear operator $d: \mathcal{H}^{\bullet} \rightarrow \mathcal{H}^{\bullet}$ such that $d^{2}=0$, which satisfies the Leibniz rule

$$
d(a b)=d(a) b+(-1)^{\operatorname{deg} a} a d(b)
$$

for all $a, b \in \mathcal{H}^{\bullet}$ with $a$ homogeneous. A DG left $\mathcal{H}^{\bullet}$-module is a $\mathbb{Z}$-graded $\mathcal{H}^{\bullet}$ module $M^{\bullet}=\bigoplus_{i \in \mathbb{Z}} M^{i}$ with a $k$-linear differential $d: M^{\bullet} \rightarrow M^{\bullet}$ of degree one which satisfies an analogous Leibniz rule. In this situation the cohomology algebra $h^{*}\left(\mathcal{H}^{\bullet}\right)=\bigoplus_{i \in \mathbb{Z}} h^{i}\left(\mathcal{H}^{\bullet}\right)$ is a graded algebra, and $h^{*}\left(M^{\bullet}\right)=\bigoplus_{i \in \mathbb{Z}} h^{i}\left(M^{\bullet}\right)$ is a graded (left) module over $h^{*}\left(\mathcal{H}^{\bullet}\right)$.

We follow the conventions of [BL94, p. 68] regarding DGA's and DG-modules all being unital. More precisely we always assume the existence of a multiplicative identity $1 \in \mathcal{H}^{0}$ such that $d(1)=0$. Then $h^{*}\left(\mathcal{H}^{\bullet}\right)$ inherits a multiplicative identity $1 \in h^{0}\left(\mathcal{H}^{\bullet}\right)$. Moreover we assume that $1 \in \mathcal{H}^{0}$ acts trivially on all our DG-modules $M^{\bullet}$.

Remark 4.1. Our main examples of DG algebras and modules will arise as follows. Let $\mathcal{A}$ be an abelian category and consider two (unbounded) complexes $I^{\bullet}$ and $J^{\bullet}$ in $C(\mathcal{A})$. The associated morphism complex $\operatorname{Hom}_{\mathcal{A}}^{\bullet}\left(I^{\bullet}, J^{\bullet}\right)$ of abelian groups has $i$ th component (cf. [Har66, p. 63])

$$
\operatorname{Hom}_{\mathcal{A}}^{i}\left(I^{\bullet}, J^{\bullet}\right)=\prod_{q \in \mathbb{Z}} \operatorname{Hom}_{\mathcal{A}}\left(I^{q}, J^{q+i}\right) .
$$

An element hereof is just a collection of morphisms $a=\left(a_{q}\right)_{q \in \mathbb{Z}}$ where each $a_{q}$ shifts the degrees by $i$ (there is no compatibility requirement with the differentials). The differentials are defined below:

$$
\operatorname{Hom}_{\mathcal{A}}^{i}\left(I^{\bullet}, J^{\bullet}\right) \stackrel{d}{\longrightarrow} \operatorname{Hom}_{\mathcal{A}}^{i+1}\left(I^{\bullet}, J^{\bullet}\right) \quad d(a)_{q}=d \circ a_{q}-(-1)^{i} a_{q+1} \circ d .
$$

If we take the same complex $J^{\bullet}=I^{\bullet}$, the morphism complex $\operatorname{Hom}_{\mathcal{A}}^{\bullet}\left(I^{\bullet}, I^{\bullet}\right)$ carries the structure of a DGA over $\mathbb{Z}$. Two homogeneous elements $a$ and $b$, of degree $i$ and $j$, respectively, are multiplied by the rule

$$
(a b)_{q}=(-1)^{i j} a_{q+j} \circ b_{q} .
$$

When $J^{\bullet}$ is arbitrary a similar formula endows $\operatorname{Hom}_{\mathcal{A}}^{\bullet}\left(I^{\bullet}, J^{\bullet}\right)$ with the structure of a DG right module over $\operatorname{End}_{\mathcal{A}}^{\bullet}\left(I^{\bullet}\right)$ or what amounts to the same: a DG left module over the opposite $\operatorname{End}_{\mathcal{A}}^{\bullet}\left(I^{\bullet}\right)^{\text {op }}$.

It follows straight from the definition of the differentials in the morphism complex that its $i$-cycles correspond to morphisms of complexes $I^{\bullet} \rightarrow J^{\bullet+i}$ (recall that the translation functor on $D(\mathcal{A})$ also changes signs of the differentials) and its $i$-boundaries are null-homotopic; cf. [Har66, p. 64]. Consequently

$$
h^{i}\left(\operatorname{Hom}_{\mathcal{A}}^{\bullet}\left(I^{\bullet}, J^{\bullet}\right)\right)=\operatorname{Hom}_{K(\mathcal{A})}\left(I^{\bullet}, J^{\bullet+i}\right) .
$$

When $\mathcal{A}$ has enough injectives and $J^{\bullet}$ is bounded below one can replace $K(\mathcal{A})$ by the derived category above and identify the right-hand side with the $i$ th hyperext $\operatorname{Ext}_{\mathcal{A}}^{i}\left(I^{\bullet}, J^{\bullet}\right)$; cf. [Har66, Thm. 6.4].

We return to a general DGA $\mathcal{H}^{\bullet}$ over a field $k$ and the category of DG left $\mathcal{H}^{\bullet}$-modules. There is an obvious notion of quasi-isomorphism, and by localization one obtains the derived category $D\left(\mathcal{H}^{\bullet}\right)$, which naturally becomes a triangulated 
category. As for abelian categories, morphisms in the derived category are easier to work with (as equivalence classes of roofs) if one localizes the homotopy category. In the DGA setup the morphisms of $K\left(\mathcal{H}^{\bullet}\right)$ are homotopy classes of maps between DG modules, where $f: M^{\bullet} \rightarrow N^{\bullet}$ is null-homotopic if there is a map $r: M^{\bullet} \rightarrow N^{\bullet-1}$ of graded $\mathcal{H}^{\bullet}$-modules for which $f=d \circ r+r \circ d$. A streamlined exposition of these constructions is in BL94, Ch. 10].

Keller proved that the triangulated categories equivalent to some $D\left(\mathcal{H}^{\bullet}\right)$ can be characterized by a short list of properties. First, $D\left(\mathcal{H}^{\bullet}\right)$ is always algebraic. Recall that a triangulated category $\mathcal{D}$ is said to be algebraic if it is equivalent to the stable category of a Frobenius category. We will not recall these notions here but just refer to [Kra07, Ch. 7], where this definition is discussed in great detail. What we will use later is the following simple criterion of Krause; see [Kra07, Lem. 7.5].

Lemma 4.2. A triangulated category $\mathcal{D}$ is algebraic if and only if there is an additive category $\mathcal{A}$ and a fully faithful exact functor $\mathcal{D} \longrightarrow K(\mathcal{A})$.

Secondly, $D\left(\mathcal{H}^{\bullet}\right)$ admits (set-indexed) coproducts which can be computed as direct sums of DG $\mathcal{H}^{\bullet}$-modules, and the module $\mathcal{H}^{\bullet}$ is a compact generator. Recall that when $\mathcal{D}$ admits coproducts, an object $C \in \operatorname{Ob}(\mathcal{D})$ is said to be compact if the functor $\operatorname{Hom}_{\mathcal{D}}(C,-)$ commutes with coproducts. We say that $C$ generates $\mathcal{D}$ if $X=0$ precisely when $\operatorname{Hom}_{\mathcal{D}}\left(C, T^{i} X\right)=0$ for all $i \in \mathbb{Z}$.

Keller's Morita theorem shows that these properties characterize the categories $D\left(\mathcal{H}^{\bullet}\right)$.

Theorem 4.3. Let $\mathcal{D}$ be an algebraic triangulated category which admits arbitrary coproducts and a compact generator $C \in O b(\mathcal{D})$. Then there are a differential graded algebra $\mathcal{H}_{C}^{\bullet}=R \operatorname{Hom}_{\mathcal{D}}(C, C)^{\mathrm{op}}$ and an equivalence of triangulated categories $\mathcal{D} \longrightarrow D\left(\mathcal{H}_{C}^{\bullet}\right)$.

Proof. This is part of [Kel07, Sec. 8.7, Thm. 8.5(b)] (using a different notation).

In the theorem $R \operatorname{Hom}_{\mathcal{D}}(C, C)$ is a certain complex with $i$ th cohomology $\operatorname{Hom}_{\mathcal{D}}\left(C, T^{i} C\right)$. We will apply the theorem only to situations where $\mathcal{D}=D(\mathcal{A})$ for some abelian category $\mathcal{A}$ with enough injectives and the compact generator is an object $C$ of $\mathcal{A}$ thought of as the complex $C[0]$ concentrated in degree zero. Upon choosing an injective resolution $C[0] \rightarrow I^{\bullet}$ the associated DGA is $\mathcal{H}^{\bullet}=\operatorname{End}_{\mathcal{A}}^{\bullet}\left(I^{\bullet}\right)^{\text {op }}$ as defined in Remark 4.1. In this situation the functor $D(\mathcal{A}) \rightarrow D\left(\mathcal{H}^{\bullet}\right)$ from Theorem 4.3 is the formation of the morphism complex $\operatorname{Hom}_{\mathcal{A}}^{\bullet}\left(I^{\bullet},-\right)$ as above after taking a $K$-injective resolution.

The cohomology algebra $h^{*}\left(\mathcal{H}^{\bullet}\right)$ has the following nice description in terms of the Yoneda algebra:

$$
h^{*}\left(\mathcal{H}^{\bullet}\right)=\operatorname{Ext}_{\mathcal{A}}^{*}(C, C)^{\mathrm{op}}=\bigoplus_{i \geq 0} \operatorname{Ext}_{\mathcal{A}}^{i}(C, C) .
$$

Similarly for the cohomology of $\operatorname{Hom}_{\mathcal{A}}^{\bullet}\left(I^{\bullet},-\right)$; cf. [Har66, Cor. 6.5], which compares Ext $^{i}$ to hyperext.

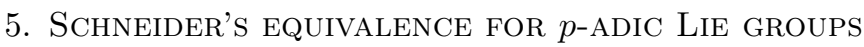

In this section we assume $k$ is a field of characteristic $p>0$ and that $G$ is a torsionfree pro- $p$ group. Moreover, we will assume that $G$ is a $p$-adic Lie group of dimension $d=\operatorname{dim}(G)$ over $\mathbb{Q}_{p}$, say. 
By a result of Serre such $G$ have finite cohomological $p$-dimension $\operatorname{cd}_{p}(G)=d$; cf. Ser65, Cor. (1)]. In that paper Serre also mentions that the $p$-dimension $\mathrm{cd}_{p}$ is infinite if there are elements of order $p$. We will need this finiteness result to guarantee that certain derived functors are defined on all of $D(G)$.

Furthermore, our assumptions on $G$ ensure that $\Omega=k \llbracket G \rrbracket$ is a Noetherian ring which is local with maximal ideal $\mathfrak{m}=\operatorname{ker}(a)$ since $G$ is pro- $p$. In particular the topology on $\Omega$, which is defined by the ideals $I_{N}=\operatorname{ker}(\Omega \rightarrow k[G / N])$, coincides with the m-adic topology. See [Sch11, Prop. 19.7, Lem. 19.8, Thm. 33.4] for these standard results about $p$-adic Lie groups.

Schneider's idea in Sch15] was to apply Keller's Theorem 4.3 to $D(G)$. In fact Schneider works in a more general setup with a pair $(G, I)$ where $G$ is any $p$-adic Lie group (not necessarily compact) and $I \subset G$ is a compact open subgroup which is torsionfree and pro- $p$. Here we will be interested only in the case $G=I$. The results in this section are entirely due to Schneider. We hope our exposition will help the reader to quickly grasp the overall strategy behind [Sch15].

To apply Theorem 4.3 we first have to observe that $D(G)$ is an algebraic triangulated category.

Lemma 5.1 (Sch15, Lem. 8]). D $(G)$ is algebraic 2

Proof. Using Krause's Lemma 4.2 this follows rather immediately from the existence of $K$-injective resolutions. Recall that a complex $V^{\bullet}$ is said to be $K$-injective if

$$
\operatorname{Hom}_{K(G)}\left(U^{\bullet}, V^{\bullet}\right) \stackrel{\sim}{\longrightarrow} \operatorname{Hom}_{D(G)}\left(U^{\bullet}, V^{\bullet}\right)
$$

for all complexes $U^{\bullet}$ (see [Spa88, Prop. 1.5]). Since $\operatorname{Rep}_{k}^{\infty}(G)$ is a Grothendieck category (meaning filtered colimits are exact and it has a generator $Y$ ) every complex $V^{\bullet}$ has a $K$-injective resolution; cf. [Sta18, Tag 079P, Thm. 19.12.6]. That is, there is a $K$-injective complex $I^{\bullet}$ and a quasi-isomorphism $V^{\bullet} \rightarrow I^{\bullet}$. The $K$-injective complexes form a full triangulated subcategory $K_{\text {inj }}(G)$ of the homotopy category (see [Spa88, Prop. 1.3]), and there is a triangle equivalence

$$
K_{\mathrm{inj}}(G) \stackrel{\sim}{\longrightarrow} D(G) .
$$

Once and for all we fix a quasi-inverse i. This shows that there is a fully faithful exact functor $D(G) \rightarrow K(G)$ and therefore $D(G)$ is algebraic by Lemma 4.2 .

It is easy to see that $D(G)$ has arbitrary coproducts. They are just direct sums of complexes; cf. [Sch15, Rem. 2]. The next step is to show that the trivial representation is a compact generator for $D(G)$. Occasionally when we write $k$ below we mean the trivial representation $G \stackrel{1}{\longrightarrow} k^{\times}$concentrated in degree 0 . When there is a risk of confusion we will write $k[0]$ instead.

Lemma 5.2 ([Sch15, Lem. 4]). The trivial representation $k$ is a compact object of $D(G)$. That is, the natural map

$$
\bigoplus_{j \in J} \operatorname{Hom}_{D(G)}\left(k, V_{j}^{\bullet}\right) \stackrel{\sim}{\longrightarrow} \operatorname{Hom}_{D(G)}\left(k, \bigoplus_{j \in J} V_{j}^{\bullet}\right)
$$

is an isomorphism of abelian groups for any collection of complexes $\left(V_{j}^{\bullet}\right)_{j \in J}$.

\footnotetext{
${ }^{2}$ The same proof shows that the derived category of any Grothendieck category is algebraic.
} 
Proof. We have to show the functor $\operatorname{Hom}_{D(G)}(k,-)$ commutes with direct sums. Note that for all $V^{\bullet}$,

$$
\begin{aligned}
\operatorname{Hom}_{D(G)}\left(k, V^{\bullet}\right) & =\operatorname{Hom}_{D(G)}\left(k, \mathbf{i}\left(V^{\bullet}\right)\right) \\
& =\operatorname{Hom}_{K(G)}\left(k, \mathbf{i}\left(V^{\bullet}\right)\right) \\
& =h^{0}\left(\operatorname{Hom}_{G}^{\bullet}\left(k[0], \mathbf{i}\left(V^{\bullet}\right)\right)\right) \\
& =h^{0}\left(\operatorname{Hom}_{G}\left(k, \mathbf{i}\left(V^{\bullet}\right)\right)\right) \\
& =h^{0}\left(\mathbf{i}\left(V^{\bullet}\right)^{G}\right) \\
& =h^{0}\left(G, V^{\bullet}\right) .
\end{aligned}
$$

Here $h^{i}\left(G, V^{\bullet}\right)$ denotes group hypercohomology, by which we mean the following. Consider the additive functor $\Gamma: \operatorname{Rep}_{k}^{\infty}(G) \rightarrow \operatorname{Vec}_{k}$ taking $G$-invariants $(\cdot)^{G}$. Since $R^{i} \Gamma=0$ for $i>d=\operatorname{cd}_{p}(G)$ the total derived functor $R \Gamma$ is defined on all of $D(G)$ by Har66, Cor. $5.3(\gamma)$ ] and is given by the composition

$$
D(G) \stackrel{\mathbf{i}}{\longrightarrow} K_{\mathrm{inj}}(G) \longrightarrow K\left(\mathrm{Vec}_{k}\right) \longrightarrow D\left(\mathrm{Vec}_{k}\right),
$$

which takes $V^{\bullet} \mapsto \mathbf{i}\left(V^{\bullet}\right)^{G}$. Group hypercohomology is then $h^{i}\left(G, V^{\bullet}\right)=h^{i}\left(R \Gamma\left(V^{\bullet}\right)\right)$. Note that negative $i$ are allowed here. Since (actual) group cohomology commutes with direct sums in $\operatorname{Rep}_{k}^{\infty}(G)$ a direct sum of $\Gamma$-acyclic objects is $\Gamma$-acyclic. After choosing quasi-isomorphisms $V_{j}^{\bullet} \rightarrow C_{j}^{\bullet}$ with complexes having $\Gamma$-acyclic terms it is then easy to see that

$$
\bigoplus_{j \in J} h^{i}\left(G, V_{j}^{\bullet}\right) \stackrel{\sim}{\longrightarrow} h^{i}\left(G, \bigoplus_{j \in J} V_{j}^{\bullet}\right) .
$$

With the observation (5.3) the case $i=0$ gives the compactness of $k$. See the proof of [Sch15, Lem. 3] for more details.

The calculation (5.3) shows more generally that, for any $V^{\bullet}$ and any integer $i \in \mathbb{Z}$, we have

$$
\operatorname{Hom}_{D(G)}\left(k, T^{i} V^{\bullet}\right)=h^{i}\left(G, V^{\bullet}\right) .
$$

The most difficult part of Sch15] is the proof that $k$ is a generator of $D(G)$. We find it worthwhile to paraphrase Schneider's elegant proof below; our rendition will hopefully be helpful.

Proposition 5.4 ([Sch15, Prop. 5]). The trivial representation $k$ is a generator of $D(G)$. That is,

$$
\operatorname{Hom}_{D(G)}\left(k, T^{i} V^{\bullet}\right)=0 \quad \forall i \in \mathbb{Z} \Longrightarrow V^{\bullet}=0 .
$$

Proof. Our assumption that $h^{i}\left(G, V^{\bullet}\right)=0$ for all $i \in \mathbb{Z}$ means that $R \Gamma\left(V^{\bullet}\right)$ is exact. We have to conclude that $V^{\bullet}$ is exact. We will introduce a sequence of functors $\Gamma_{j}$ starting from $\Gamma_{1}=\Gamma$ which all admit total derived functors $R \Gamma_{j}$ defined on $D(G)$. By induction we will show that $R \Gamma_{j}\left(V^{\bullet}\right)$ is exact for all $j \in \mathbb{N}$. If the terms of $V^{\bullet}$ are $\Gamma_{j}$-acyclic this means that $\Gamma_{j}\left(V^{\bullet}\right)$ is exact and we would be able to conclude that $V^{\bullet}=\lim _{j \in \mathbb{N}} \Gamma_{j}\left(V^{\bullet}\right)$ is exact.

The forthcoming definition of the functors $\Gamma_{j}$ is based on the following observation. We first note that $\Omega / I_{N} \simeq \operatorname{ind}_{N}^{G}(1)$ is an object of $\operatorname{Rep}_{k}^{\infty}(G)$ and $\operatorname{Hom}_{G}\left(\Omega / I_{N}, V\right)=V^{N}$ for any smooth representation $V$ by Frobenius reciprocity. 
Now since $\mathfrak{m}^{j}$ is open it contains $I_{N}$ for small enough $N$, and therefore $\Omega / \mathfrak{m}^{j}$ is also an object of $\operatorname{Rep}_{k}^{\infty}(G)$. We define the functors $\Gamma_{j}: \operatorname{Rep}_{k}^{\infty}(G) \rightarrow \operatorname{Vec}_{k}$ by

$$
\Gamma_{j}(V)=\operatorname{Hom}_{G}\left(\Omega / \mathfrak{m}^{j}, V\right) .
$$

Note that $\Gamma_{1}=\Gamma$. We may identify $\Gamma_{j}(V)$ with a subspace of $V^{N}$ when $\mathfrak{m}^{j} \supset I_{N}$. Since the powers $\mathfrak{m}^{j}$ form a fundamental system of open neighborhoods at zero in $\Omega$, smoothness of $V$ translates into

$$
V=\bigcup_{j \in \mathbb{N}} \Gamma_{j}(V)
$$

In order to show that $R \Gamma_{j}$ is defined on all of $D(G)$ it suffices (again by Har66, Cor. 5.3( $\gamma)]$ ) to show that

$$
R^{i} \Gamma_{j}(V)=\operatorname{Ext}_{G}^{i}\left(\Omega / \mathfrak{m}^{j}, V\right)=0 \quad \forall i>d .
$$

Since $\Omega$ is Noetherian $\mathfrak{m}^{j} / \mathfrak{m}^{j+1} \simeq k^{\oplus n_{j}}$ for some $n_{j} \in \mathbb{N}$ (with $G$ acting trivially on both sides of the isomorphism). The short exact sequence of smooth $G$ representations

$$
0 \longrightarrow \mathfrak{m}^{j} / \mathfrak{m}^{j+1} \longrightarrow \Omega / \mathfrak{m}^{j+1} \longrightarrow \Omega / \mathfrak{m}^{j} \longrightarrow 0
$$

gives rise to a long exact sequence of $k$-vector spaces

$$
\begin{aligned}
\cdots & \longrightarrow \operatorname{Ext}_{G}^{i}\left(\Omega / \mathfrak{m}^{j}, V\right) \longrightarrow \operatorname{Ext}_{G}^{i}\left(\Omega / \mathfrak{m}^{j+1}, V\right) \longrightarrow \operatorname{Ext}_{G}^{i}(k, V)^{\oplus n_{j}} \\
& \longrightarrow \operatorname{Ext}_{G}^{i+1}\left(\Omega / \mathfrak{m}^{j}, V\right) \longrightarrow \cdots,
\end{aligned}
$$

from which (5.5) follows immediately by induction on $j$. The long exact sequence also shows that if $V$ is $\Gamma$-acyclic, then $V$ is $\Gamma_{j}$-acyclic for all $j \in \mathbb{N}$.

We proceed to show that $R \Gamma_{j}\left(V^{\bullet}\right)$ is exact (the case $j=1$ being our assumption on $\left.V^{\bullet}\right)$. The analogous long exact sequence for hyperext functors is

$$
\begin{aligned}
\cdots & \longrightarrow \operatorname{Ext}_{G}^{i}\left(\Omega / \mathfrak{m}^{j}, V^{\bullet}\right) \longrightarrow \operatorname{Ext}_{G}^{i}\left(\Omega / \mathfrak{m}^{j+1}, V^{\bullet}\right) \longrightarrow \operatorname{Ext}_{G}^{i}\left(k, V^{\bullet}\right)^{\oplus n_{j}} \\
& \longrightarrow \operatorname{Ext}_{G}^{i+1}\left(\Omega / \mathfrak{m}^{j}, V^{\bullet}\right) \longrightarrow \cdots ;
\end{aligned}
$$

see [Har66, Prop. 6.1]. By induction all terms here vanish. A calculation similar to (5.3) shows that

$$
\operatorname{Ext}_{G}^{i}\left(\Omega / \mathfrak{m}^{j}, V^{\bullet}\right):=\operatorname{Hom}_{D(G)}\left(\Omega / \mathfrak{m}^{j}, T^{i} V^{\bullet}\right)=h^{i}\left(R \Gamma_{j}\left(V^{\bullet}\right)\right) .
$$

We may assume all terms of $V^{\bullet}$ are $\Gamma$-acyclic and therefore $\Gamma_{j}$-acyclic as observed. As a result $\Gamma_{j}\left(V^{\bullet}\right)$ is exact. Their colimit over $j$ equals $V^{\bullet}$, which is therefore exact.

Using results of Neeman, Schneider deduces that $k$ generates $D(G)$ in the sense that any strict full triangulated subcategory $\mathcal{D} \subset D(G)$, closed under direct sums, which contains $k$ must be all of $D(G)$; cf. [Sch15, Prop. 6].

By contrast $k$ does not generate $\operatorname{Rep}_{k}^{\infty}(G)$ as an abelian category. It generates the subcategory of representations $V$ which are generated by $V^{G}$. Note that $V^{G} \neq 0$ for non-zero $V$ (pick a non-zero $v \in V$ and decompose the finite set $\mathbb{F}_{p}[G] v$ into $G$-orbits).

Combining the previous three results (Lemmas 5.1 and 5.2. and Proposition 5.4), Keller's Theorem 4.3 implies the main result of [Sch15] in our setup $(G=I)$ :

Theorem 5.6 ([Sch15, Thm. 9]). There is an equivalence of triangulated categories

$$
D(G) \stackrel{\sim}{\longrightarrow} D\left(\mathcal{H}^{\bullet}\right) .
$$


Here $\mathcal{H}^{\bullet}=\operatorname{End}_{G}^{\bullet}\left(I^{\bullet}\right)^{\text {op }}$ for a choice of injective resolution $k[0] \rightarrow I^{\bullet}$ (which one can obviously take to be non-negatively graded), and the equivalence is given by the composition

$$
H: D(G) \stackrel{\text { i }}{\longrightarrow} K_{\text {inj }}(G) \longrightarrow K\left(\mathcal{H}^{\bullet}\right) \longrightarrow D\left(\mathcal{H}^{\bullet}\right) \quad V^{\bullet} \rightsquigarrow \operatorname{Hom}_{G}^{\bullet}\left(I^{\bullet}, \mathbf{i}\left(V^{\bullet}\right)\right) .
$$

A quasi-inverse can be defined in a similar fashion. Following BL94, Def. 10.12.2.1] we call a DG module $M^{\bullet} K$-projective if the natural map $\operatorname{Hom}_{K(\mathcal{H} \bullet}\left(M^{\bullet}, N^{\bullet}\right) \longrightarrow$ $\operatorname{Hom}_{D(\mathcal{H} \bullet)}\left(M^{\bullet}, N^{\bullet}\right)$ is an isomorphism for all other DG modules $N^{\bullet}$. By [BL94, Cor. 10.12.2.9] localization restricts to an equivalence of triangulated categories $K_{\text {pro }}\left(\mathcal{H}^{\bullet}\right) \longrightarrow D\left(\mathcal{H}^{\bullet}\right)$. We fix a quasi-inverse $\mathbf{p}$ once and for all and let $T$ be the composition

$$
T: D\left(\mathcal{H}^{\bullet}\right) \stackrel{\mathbf{p}}{\longrightarrow} K_{\text {pro }}\left(\mathcal{H}^{\bullet}\right) \longrightarrow K(G) \longrightarrow D(G) \quad M^{\bullet} \rightsquigarrow I^{\bullet} \otimes_{\mathcal{H}} \bullet \mathbf{p}\left(M^{\bullet}\right) .
$$

Here we view $I^{\bullet}$ as the right DG $\mathcal{H}^{\bullet}$-module $\bigoplus_{i>0} I^{i}$. The tensor product is defined in [BL94, 10.9]. This functor $T$ is both a left adjoint to $H$ and a quasi-inverse of $H$.

As noted after Theorem 4.3 the cohomology algebra is $h^{*}\left(\mathcal{H}^{\bullet}\right)=\operatorname{Ext}_{G}^{*}(k, k)^{\mathrm{op}}$. Moreover,

$$
h^{*}\left(H\left(V^{\bullet}\right)\right)=\bigoplus_{i \in \mathbb{Z}} \operatorname{Ext}_{G}^{i}\left(k, V^{\bullet}\right)
$$

for any complex of smooth $G$-representations $V^{\bullet}$.

\section{The Yoneda Algebra of $\Omega$}

We defined the DGA $\mathcal{H}^{\bullet}=\operatorname{End}_{G}^{\bullet}\left(I^{\bullet}\right)^{\text {op }}$ by choosing an injective resolution of the trivial representation, say $k[0] \rightarrow I^{\bullet}$. By Pontryagin duality this corresponds to choosing a projective resolution $P^{\bullet} \rightarrow k[0]$ in the category $\operatorname{Mod}(\Omega)$ of pseudocompact modules. Here we view $k$ as a module over $\Omega$ via the augmentation map $\Omega \stackrel{a}{\longrightarrow} k$, and $P^{\bullet}$ is indexed non-positively:

$$
\cdots \longrightarrow P^{-2} \longrightarrow P^{-1} \longrightarrow P^{0} \longrightarrow k \longrightarrow 0 .
$$

To go between the two points of view use the relation $P^{-i}=\left(I^{i}\right)^{\vee}$ for $i \geq 0$.

Lemma 6.1. We have the following isomorphisms.

(1) $\mathcal{H}^{\bullet}=\operatorname{End}_{G}^{\bullet}\left(I^{\bullet}\right)^{\text {op }} \stackrel{\sim}{\longrightarrow} \operatorname{End}_{\Omega}^{\bullet}\left(P^{\bullet}\right)$;

(2) $h^{*}\left(\mathcal{H}^{\bullet}\right)=\operatorname{Ext}_{G}^{*}(k, k)^{\mathrm{op}} \stackrel{\sim}{\longrightarrow} \operatorname{Ext}_{\Omega}^{*}(k, k)$.

Proof. Consider a homogeneous element $a=\left(a_{q}\right)_{q \in \mathbb{Z}}$ in $\operatorname{End}_{G}^{i}\left(I^{\bullet}\right)$. Here $a_{q}: I^{q} \rightarrow$ $I^{q+i}$ has Pontryagin dual $a_{q}^{\vee}: P^{-q-i} \rightarrow P^{-q}$. Introducing $t=-q-i$ this is a map $b_{t}: P^{t} \rightarrow P^{t+i}$. Packaged together they give an element $b=\left(b_{t}\right)_{t \in \mathbb{Z}}$ in $\operatorname{End}_{\Omega}^{i}\left(P^{\bullet}\right)$. To summarize, $b_{t}=a_{-t-i}^{\vee}$. Since $(\cdot)^{\vee}$ is an anti-equivalence, composition on $\operatorname{End}_{\Omega}^{\bullet}\left(P^{\bullet}\right)$ corresponds to opposite composition on $\operatorname{End}_{G}^{\bullet}\left(I^{\bullet}\right)$. This proves (1), which then implies (2) by taking cohomology.

There is a canonical choice of $P^{\bullet}$ which is the bar resolution of $k$; cf. Laz65, Ch. V, (1.2.8)]. In this resolution the $P^{-i}$ are $(i+1)$-fold completed tensor products (thought of as $\Omega$-modules via the leftmost factor)

$$
P^{-i}=\Omega^{\hat{\otimes}(i+1)}=\Omega \hat{\otimes}_{k} \cdots \hat{\otimes}_{k} \Omega=\Omega(G \times \cdots \times G),
$$

and the differentials are obtained from the case of $k[G / N]$ by passing to the limit; cf. Laz65, Ch. V, (1.2.1.2)] for the explicit formula. The first differential $P^{0}=\Omega \rightarrow k$ 
is the augmentation map $a$. Dually the $I^{i}$ are direct sums of (an enormous number of) copies of the regular representation $\mathcal{C}^{\infty}(G, k)$.

In conjunction Schneider's Theorem 5.6 and Pontryagin duality (Corollary [3.2) give an equivalence

$$
D(\Omega)^{\mathrm{op}} \stackrel{\sim}{\longrightarrow} D\left(\operatorname{End}_{\Omega}^{\bullet}\left(P^{\bullet}\right)\right)
$$

which takes a $K$-projective complex $X^{\bullet}$ of pseudocompact $\Omega$-modules to the left DG $\operatorname{End}_{\Omega}^{\bullet}\left(P^{\bullet}\right)$-module $\operatorname{Hom}_{\Omega}^{\bullet}\left(X^{\bullet}, P^{\bullet}\right)$. After passing to cohomology this yields a left module $\bigoplus_{i \in \mathbb{Z}} \operatorname{Ext}_{\Omega}^{i}\left(X^{\bullet}, k\right)$ over the Yoneda algebra

$$
E(\Omega):=\operatorname{Ext}_{\Omega}^{*}(k, k) .
$$

One of our goals in this paper is to endow $E(\Omega)$ with an $A_{\infty}$-structure and give an equivalence between $D(\Omega)^{\text {op }}$ and the derived category of $A_{\infty}$-modules $D_{\infty}(E(\Omega))$ akin to Koszul duality. We pause before doing so and discuss the case of equi- $p$ valued groups $G$ where $E(\Omega)$ has a nice explicit description due to Lazard.

\section{REVIEW OF LAZARD'S THEORY OF $p$-VALUED GROUPS}

7.1. Filtrations on $p$-valued groups. Suppose our pro- $p$ group $G$ is equipped with a $p$-valuation $\omega$ defining the topology. This means the following. First recall that $\omega: G \backslash\{1\} \longrightarrow\left(\frac{1}{p-1}, \infty\right)$ is a function satisfying three axioms, one of which is that $\omega\left(g^{p}\right)=\omega(g)+1$; cf. [Sch11, p. 169]. In particular $G$ cannot have elements of order $p$. The other conditions on $\omega$ guarantee that

$$
G_{v}=\{g \in G: \omega(g) \geq v\} \quad G_{v+}=\{g \in G: \omega(g)>v\}
$$

are normal subgroups of $G$. When we say $\omega$ defines the topology on $G$ we mean that each $G_{v}$ is open and $G=\lim G / G_{v}$. We associate an abelian group $\operatorname{gr}(G)$ with the $p$-valued group $(G, \omega)$ as follows:

$$
\operatorname{gr}(G)=\bigoplus_{v>0} \operatorname{gr}_{v}(G)=\bigoplus_{v>0} G_{v} / G_{v+} .
$$

It turns out that $\operatorname{gr}(G)$ has a lot more structure. First of all it is an $\mathbb{F}_{p \text {-vector space }}$ since $g^{p} G_{v+}=1 G_{v+}$. Moreover, taking commutators defines a Lie bracket [-,-] on $\operatorname{gr}(G)$ compatible with the grading. Lastly, $\operatorname{gr}(G)$ becomes a module over the one-variable polynomial ring $\mathbb{F}_{p}[\pi]$ by letting $\pi$ act by the degree one map

$$
\pi: \operatorname{gr}(G) \longrightarrow \operatorname{gr}(G) \quad g G_{v+} \mapsto g^{p} G_{(v+1)+} .
$$

One can show that the Lie bracket is $\mathbb{F}_{p}[\pi]$-bilinear and that $\operatorname{gr}(G)$ thus becomes a graded Lie algebra over $\mathbb{F}_{p}[\pi]$. We refer to [Sch11, Secs. 23-25] for more details. If $k$ is a field of characteristic $p$ we convert $\operatorname{gr}(G)$ into a graded Lie algebra $\mathfrak{g}$ over $k$ as follows. This $\mathfrak{g}$ will play an important role below.

Definition 7.1. $\mathfrak{g}:=k \otimes_{\mathbb{F}_{p}[\pi]} \operatorname{gr}(G)=k \otimes_{\mathbb{F}_{p}} \operatorname{gr}(G) / \pi \operatorname{gr}(G)$.

In general $\operatorname{gr}(G)$ is torsionfree over $\mathbb{F}_{p}[\pi]$. Since we are assuming $G$ is a $d$ dimensional Lie group over $\mathbb{Q}_{p}$, in fact $\operatorname{gr}(G)$ is free over $\mathbb{F}_{p}[\pi]$ of rank $d$. Consequently $(G, \omega)$ admits an ordered basis, by which we mean a tuple $\left(g_{1}, \ldots, g_{d}\right)$ of elements from $G$ with the two properties below.

- The map $\left(x_{1}, \ldots, x_{d}\right) \mapsto g_{1}^{x_{1}} \cdots g_{d}^{x_{d}}$ is a homeomorphism $\mathbb{Z}_{p}^{d} \longrightarrow G$;

- $\omega(g)=\min _{i=1, \ldots, d}\left\{\omega\left(g_{i}\right)+v_{\mathbb{Z}_{p}}\left(x_{i}\right)\right\}$ where $g=g_{1}^{x_{1}} \cdots g_{d}^{x_{d}}$. 
Correspondingly the elements $\sigma\left(g_{i}\right):=g_{i} G_{\omega\left(g_{i}\right)+}$ form an $\mathbb{F}_{p}[\pi]$-basis for $\operatorname{gr}(G)$; cf. [Sch11, Prop. 26.5]. In turn the vectors $\xi_{i}:=1 \otimes \sigma\left(g_{i}\right)$ form a $k$-basis for $\mathfrak{g}$, and by the Poincaré-Birkhoff-Witt theorem the monomials $\xi_{1}^{\alpha_{1}} \cdots \xi_{d}^{\alpha_{d}}$ (for varying $\alpha_{i} \in \mathbb{Z}_{\geq 0}$ ) form a $k$-basis for the universal enveloping algebra $U(\mathfrak{g})$.

7.2. Filtrations on Iwasawa algebras. Now assume $k$ is a finit 3 field of characteristic $p$ and let $\mathcal{O}=W(k)$ be its ring of Witt vectors, a complete DVR with residue field $\mathcal{O} / p \mathcal{O}=k$. We let $\Lambda=\Lambda(G)=\mathcal{O} \llbracket G \rrbracket$ be the Iwasawa $\mathcal{O}$-algebra of $G$. Note that $\Lambda / p \Lambda=\Omega$ (the Mittag-Leffler condition is trivially satisfied since the transition maps between the various $\mathcal{O}[G / N]$ are surjective).

As an $\mathcal{O}$-module $\Lambda$ is isomorphic to $\mathcal{O} \llbracket X_{1}, \ldots, X_{d} \rrbracket$. If we let $\mathbf{b}_{i}:=g_{i}-1$ and $\mathbf{b}^{\alpha}:=\mathbf{b}_{1}^{\alpha_{1}} \cdots \mathbf{b}_{d}^{\alpha_{d}}$ for $\alpha=\left(\alpha_{i}\right) \in \mathbb{Z}_{\geq 0}^{d}$ an isomorphism is given by

$$
\mathcal{O} \llbracket X_{1}, \ldots, X_{d} \rrbracket \stackrel{\sim}{\longrightarrow} \Lambda \quad \sum_{\alpha} c_{\alpha} X^{\alpha} \mapsto \sum_{\alpha} c_{\alpha} \mathbf{b}^{\alpha},
$$

where $X^{\alpha}$ is defined in the obvious way. This leads to a valuation $\tilde{\omega}: \Lambda \backslash\{0\} \longrightarrow$ $\left(\frac{1}{p-1}, \infty\right)$ on the Iwasawa $\mathcal{O}$-algebra given by the formula

$$
\tilde{\omega}(\lambda)=\inf _{\alpha}\left\{v_{\mathbb{Z}_{p}}\left(c_{\alpha}\right)+\sum_{i=1}^{d} \alpha_{i} \omega\left(g_{i}\right)\right\} \quad \lambda=\sum_{\alpha} c_{\alpha} \mathbf{b}^{\alpha},
$$

where $v_{\mathbb{Z}_{p}}(p)=1$. Since $\omega(G \backslash\{1\})$ is a discrete subset of $(0, \infty)$ one may replace the infimum by the minimum in the definition of $\tilde{\omega}$. Analogously to $G_{v}$ and $G_{v+}$ we define $\mathcal{O}$-submodules of $\Lambda$ as

$$
\Lambda_{v}=\{\lambda \in \Lambda: \tilde{\omega}(\lambda) \geq v\} \quad \Lambda_{v+}=\{\lambda \in \Lambda: \tilde{\omega}(\lambda)>v\} .
$$

They turn out to be two-sided ideals of $\Lambda$ which form a fundamental system of open neighborhoods of zero in $\Lambda$ (as $v>0$ varies). Recall that $\Lambda$ is a local ring since $G$ is pro- $p$ and its pseudocompact topology coincides with the $\mathfrak{m}_{\Lambda}$-adic topology where $\mathfrak{m}_{\Lambda}=\left\{\lambda: v_{\mathbb{Z}_{p}}\left(c_{0}\right)>0\right\}$. Each $\Lambda_{v}$ has a more explicit description (cf. Sch11, p. 197 and Thm. 28.3(ii)]) which in particular shows that $\Lambda_{v}$ is independent of the choice of basis $\left(g_{i}\right)$ but dependent on $\omega$ of course. The same is true for $\tilde{\omega}$; $\mathrm{cf}$. Sch11, Cor. 28.4]. Moreover multiplication takes $\Lambda_{v} \times \Lambda_{v^{\prime}} \rightarrow \Lambda_{v+v^{\prime}}$, which allows us to associate a graded ring $\operatorname{gr}(\Lambda)$ defined as

$$
\operatorname{gr}(\Lambda)=\bigoplus_{v>0} \operatorname{gr}_{v}(\Lambda)=\bigoplus_{v>0} \Lambda_{v} / \Lambda_{v+} .
$$

Note that $\operatorname{gr}(\Lambda)$ is a graded algebra over

$$
\operatorname{gr}(\mathcal{O})=\bigoplus_{i \geq 0} p^{i} \mathcal{O} / p^{i+1} \mathcal{O}
$$

(When $k=\mathbb{F}_{p}$ we have an isomorphism $\mathbb{F}_{p}[\pi] \stackrel{\sim}{\longrightarrow} \operatorname{gr}\left(\mathbb{Z}_{p}\right)$ sending $\pi \mapsto p+p^{2} \mathbb{Z}_{p}$. Thus we view $\operatorname{gr}(\Lambda)$ as an $\mathbb{F}_{p}[\pi]$-module; cf. the proof of Theorem 7.2 below.) We refer to [Sch11, Sec. 28] for more details on these constructions and proofs of the facts mentioned.

One of the main results in Lazard theory identifies $\operatorname{gr}(\Lambda)$ with the universal enveloping algebra $U(\operatorname{gr}(G))$ tensored by $\operatorname{gr}(\mathcal{O})$.

\footnotetext{
${ }^{3}$ Perhaps we are being over-cautious here. Perfectness should suffice.
} 
Theorem 7.2 ([Sch11, Thm. 28.3.i]). There is an isomorphism of graded $\operatorname{gr}(\mathcal{O})$ algebras

$$
\operatorname{gr}(\mathcal{O}) \otimes_{\mathbb{F}_{p}[\pi]} U(\operatorname{gr}(G)) \stackrel{\sim}{\longrightarrow} \operatorname{gr}(\Lambda) .
$$

This isomorphism is constructed as follows. Taking $g G_{v+} \mapsto(g-1)+\Lambda_{v+}$ is a group homomorphism $\operatorname{gr}_{v}(G) \rightarrow \operatorname{gr}_{v}(\Lambda)$. Varying $v$ these add up to an $\mathbb{F}_{p}[\pi]$-linear map $\operatorname{gr}(G) \rightarrow \operatorname{gr}(\Lambda)$. One checks that the Lie bracket $[-,-]$ on $\operatorname{gr}(G)$ intertwines with taking commutators on $\operatorname{gr}(\Lambda)$ via this map, which therefore by universality extends to $U(\operatorname{gr}(G))$.

Similar results hold for $\Omega$. Its elements can be uniquely expanded as $\sum_{\alpha} c_{\alpha} \mathbf{b}^{\alpha}$ now with coefficients $c_{\alpha} \in k$ of course. We define $\Omega_{v}$ to be the image of $\Lambda_{v}$ under the projection $\Lambda \rightarrow \Omega$. Similarly for $\Omega_{v+}$,

$$
\operatorname{gr}(\Omega):=\bigoplus_{v>0} \operatorname{gr}_{v}(\Omega)=\bigoplus_{v>0} \Omega_{v} / \Omega_{v+}
$$

is then a graded $k$-algebra. Observe that $\operatorname{gr}(\mathcal{O})=k \oplus\left(p \mathcal{O} / p^{2} \mathcal{O}\right) \oplus \cdots$ has a natural augmentation map $\operatorname{gr}(\mathcal{O}) \rightarrow k$, whose kernel we will denote by $\operatorname{gr}_{+}(\mathcal{O})$.

Corollary 7.3. There is an isomorphism of graded $k$-algebras

$$
U(\mathfrak{g})=k \otimes_{\mathbb{F}_{p}[\pi]} U(\operatorname{gr}(G)) \stackrel{\sim}{\longrightarrow} \operatorname{gr}(\Omega) .
$$

Proof. This follows immediately from Theorem 7.2 once we check that $k \otimes_{\operatorname{gr}(\mathcal{O})}$ $\operatorname{gr}(\Lambda) \stackrel{\sim}{\longrightarrow} \operatorname{gr}(\Omega)$. The projection $\gamma: \Lambda \rightarrow \Omega$ has kernel $p \Lambda$ and induces surjections $\Lambda_{v} \rightarrow \Omega_{v}$ (and similarly for the + versions). We get an induced map $\bar{\gamma}: \operatorname{gr}(\Lambda) \rightarrow$ $\operatorname{gr}(\Omega)$ which is clearly trivial on $\operatorname{gr}_{+}(\mathcal{O}) \cdot \operatorname{gr}(\Lambda)$. Indeed $\forall i>0$

$$
\bar{\gamma}\left(\left(p^{i} c+p^{i+1} \mathcal{O}\right)\left(\lambda+\Lambda_{v+}\right)\right)=\bar{\gamma}\left(p^{i} c \lambda+\Lambda_{(i+v)+}\right)=\gamma\left(p^{i} c \lambda\right)+\Omega_{(i+v)+}=0 .
$$

One can easily reverse this argument and show that the factored map $k \otimes_{\operatorname{gr}(\mathcal{O})}$ $\operatorname{gr}(\Lambda) \rightarrow \operatorname{gr}(\Omega)$ is injective. For instance, in the homogeneous case, if $\bar{\gamma}\left(\lambda+\Lambda_{v+}\right)=0$, then $\gamma(\lambda) \in \Omega_{v+}$, which means $\gamma(\lambda)=\gamma\left(\lambda^{\prime}\right)$ for some $\lambda^{\prime} \in \Lambda_{v+}$. Since $\operatorname{ker}(\gamma)=p \Lambda$ we infer that $\lambda=p \eta+\lambda^{\prime}$ for some $\eta \in \Lambda_{v-1}$.

Unwinding the definitions shows that the isomorphism in Corollary 7.3 sends $\xi_{i} \mapsto \mathbf{b}_{i}+\Omega_{\omega\left(g_{i}\right)+}$. More generally it takes

$$
\xi^{\alpha} \mapsto \mathbf{b}^{\alpha}+\Omega_{\left(\sum_{i=1}^{d} \alpha_{i} \omega\left(g_{i}\right)\right)+},
$$

where $\xi^{\alpha}:=\xi_{1}^{\alpha_{1}} \cdots \xi_{d}^{\alpha_{d}}$ is the PBW basis for $U(\mathfrak{g})$ introduced earlier. The grading $U(\mathfrak{g})=\bigoplus_{v>0} \operatorname{gr}_{v} U(\mathfrak{g})$ implicit in Corollary 7.3 is defined by taking $\operatorname{gr}_{v} U(\mathfrak{g})$ to be the $k$-span of all $\xi^{\alpha}$ for which $\sum_{i=1}^{d} \alpha_{i} \omega\left(g_{i}\right)=v$.

We will mostly be interested in the case where $\mathfrak{g}$ is an abelian Lie algebra. If so, then $U(\mathfrak{g})$ is the symmetric algebra $S(\mathfrak{g})$ and $\operatorname{gr}(\Omega)$ is a polynomial algebra $k\left[X_{1}, \ldots, X_{d}\right]$ with grading determined by decreeing that $\operatorname{deg}\left(X_{i}\right)=\omega\left(g_{i}\right)$ for all $i$. If these are all one this is of course the usual polynomial degree.

\section{Mod $p$ COHOMOLOGY OF EQUI- $p$-VALUED GROUPS}

We say $(G, \omega)$ is equi- $p$-valued if it admits an ordered basis $\left(g_{i}\right)$ all of whose elements have the same valuation $\omega\left(g_{i}\right)=t$. We will often assume $(G, \omega)$ is saturated (meaning all $g \in G$ with $\omega(g)>\frac{p}{p-1}$ are $p$-powers). This happens precisely when $t \leq \frac{p}{p-1}$; cf. [Sch11, Prop. 26.11]. In this case, when $p>2$ one can replace $\omega$ with $\omega^{\prime}=\omega+1-t$ and arrange for $\omega^{\prime}\left(g_{i}\right)=1$ to hold for all $i$. In other words, if $p>2$ we 
may often assume that $t=1$. (We need $p>2$ for $\frac{1}{p-1}<1$ to hold.). See Weigel's remarks in [HKN11, App. A] for more details on the switch from $\omega$ to $\omega^{\prime}$.

When $(G, \omega)$ is equi- $p$-valued the $\mathbb{F}_{p}[\pi]$-basis elements $\sigma\left(g_{i}\right)=g_{i} G_{t+}$ all lie in $\operatorname{gr}_{t}(G)$. In particular $\operatorname{gr}_{t}(G)$ generates $\operatorname{gr}(G)$ as an $\mathbb{F}_{p}[\pi]$-module. Consequently $\mathfrak{g}=\bigoplus_{v>0} \mathfrak{g}_{v}=\mathfrak{g}_{t}$ is concentrated in degree $t$ and is therefore an abelian Lie algebra, $\left[\mathfrak{g}_{t}, \mathfrak{g}_{t}\right] \subset \mathfrak{g}_{2 t}=0$. In particular $\operatorname{gr}(\Omega) \simeq S(\mathfrak{g})$ is a symmetric algebra. Having fixed the basis $\left(\xi_{i}\right)$ we may identify $S(\mathfrak{g}) \simeq k\left[X_{1}, \ldots, X_{d}\right]$ but with each $X_{i}$ of degree $t$.

Remark 8.1. A good supply of examples arises as follows. Let $F / \mathbb{Q}_{p}$ be a finite extension with integers $\mathcal{O}_{F}$ and residue field $k_{F}=\mathcal{O}_{F} / \mathfrak{m}_{F}$. We let $v_{\mathbb{Z}_{p}}$ be the valuation on $F$ with $v_{\mathbb{Z}_{p}}(p)=1$. An $n$-variable formal group law $\mathcal{F}(\underline{X}, \underline{Y})$ over $\mathcal{O}_{F}$ gives a group structure on $\mathfrak{m}_{F}^{n}=\mathfrak{m}_{F} \times \cdots \times \mathfrak{m}_{F}$ ( $n$ factors). We denote the resulting group by $\tilde{G}$ (or $\tilde{G}_{\mathcal{F}}$ if need should arise to emphasize the formal group law). These are known as Serre's standard groups. We define a function $\omega: \tilde{G} \backslash\{1\} \rightarrow(0, \infty)$ by

$$
\omega\left(x_{1}, \ldots, x_{n}\right)=\min _{i=1, \ldots, n} v_{\mathbb{Z}_{p}}\left(x_{i}\right) .
$$

This is of course not a $p$-valuation on $\tilde{G}$ itself unless $e\left(F / \mathbb{Q}_{p}\right)<p-1$, but one can show (cf. [HKN11, Lem. 2.2.2]) that $\omega$ does define a $p$-valuation on the normal subgroup

$$
G=\left\{g \in \tilde{G}: \omega(g)>\frac{1}{p-1}\right\} .
$$

Moreover $(G, \omega)$ is saturated, of rank $d=n\left[F: \mathbb{Q}_{p}\right]$, and equi-p-valued if and only if $F / \mathbb{Q}_{p}$ is unramified, in which case a $\mathbb{Z}_{p}$-basis for $\mathcal{O}_{F}$ yields an ordered basis for $G$ whose elements all have valuation $t=1$.

For instance one can start from a smooth affine group scheme $\mathcal{G}_{/ \mathcal{O}_{F}}$ of finite type and consider its formal completion $\hat{\mathcal{G}}$ along the unit $\operatorname{Spec}\left(\mathcal{O}_{F}\right) \rightarrow \mathcal{G}$. This is a smooth formal group $\hat{\mathcal{G}} \simeq \operatorname{Spf}\left(\mathcal{O}_{F} \llbracket X_{1}, \ldots, X_{n} \rrbracket\right)$; see [Dem86, p. 41]. Therefore $\hat{\mathcal{G}}$ defines an $n$-variable formal group law $\mathcal{F}(\underline{X}, \underline{Y})$ over $\mathcal{O}_{F}$ to which we can associate a $p$-valued group $(G, \omega)$ as in the previous paragraph. Unwinding the definitions it is easy to check that

$$
\tilde{G} \simeq \operatorname{ker}\left(\mathcal{G}\left(\mathcal{O}_{F}\right) \longrightarrow \mathcal{G}\left(k_{F}\right)\right) .
$$

Note that $\tilde{G}=G$ when $e\left(F / \mathbb{Q}_{p}\right)=1$ and $p>2$.

On the negative side one can show that when $p>3$ there is no way to equip $1+\mathfrak{m}_{D}$ with a $p$-valuation for which it becomes equi-p-valued. Here $D / \mathbb{Q}_{p}$ is the division quaternion algebra and $\mathfrak{m}_{D} \subset \mathcal{O}_{D}$ the maximal two-sided ideal of the maximal order. Indeed one can compute the Betti numbers $\operatorname{dim}_{\mathbb{F}_{p}} H^{i}\left(1+\mathfrak{m}_{D}, \mathbb{F}_{p}\right)$ to be $(1,3,4,3,1)$, which is not a row of Pascal's triangle; cf. Theorem 8.2 below. We found this example in [HKN11, Ex. 3.2.1]. See also [Sor19, Sec. 6.3] for an alternative calculation of the $\bmod p$ cohomology ring of $1+\mathfrak{m}_{D}$.

The following important theorem is due to Lazard.

Theorem 8.2 (Laz65, Ch. V, Prop. 2.5.7.1]). Let $(G, \omega)$ be an equi-p-valued group of finite rank. Then there is a natural isomorphism of graded $\mathbb{F}_{p}$-algebras

$$
H^{*}\left(G, \mathbb{F}_{p}\right) \stackrel{\sim}{\longrightarrow} \bigwedge \operatorname{Hom}\left(G, \mathbb{F}_{p}\right) .
$$


We outline some of the key steps in the proof. Lazard starts from the CartanEilenberg resolution $Y_{\bullet}$ of the trivial $U(\operatorname{gr}(G))$-module $\operatorname{gr}\left(\mathbb{Z}_{p}\right)=\mathbb{F}_{p}[\pi]$; cf. CE99, Thm. 7.1]:

$$
\cdots \longrightarrow U(\operatorname{gr}(G)) \otimes \bigwedge^{2} \operatorname{gr}(G) \longrightarrow U(\operatorname{gr}(G)) \otimes \bigwedge^{1} \operatorname{gr}(G) \longrightarrow U(\operatorname{gr}(G)) \longrightarrow \mathbb{F}_{p}[\pi] \longrightarrow 0 .
$$

By a lemma of Serre one can lift $Y_{\bullet}$ to a complex $X_{\bullet}$ of $\mathbb{Z}_{p} \llbracket G \rrbracket$-modules, in the sense that $\operatorname{gr} X_{\bullet}=Y_{\bullet}$. It turns out that $X_{\bullet}$ is in fact a free resolution of $\mathbb{Z}_{p}$, which Lazard dubs the quasi-minimal resolution; cf. [Laz65]. For equi- $p$-valued groups $(G, \omega)$ the quasi-minimal resolution is indeed minimal, meaning the differentials of $\mathbb{F}_{p} \otimes X_{\bullet}$ all vanish. In particular this allows one to immediately compute the Betti numbers $\operatorname{dim}_{\mathbb{F}_{p}} H^{i}\left(G, \mathbb{F}_{p}\right)=\left(\begin{array}{c}d \\ i\end{array}\right)$ where $d=\operatorname{rk}(G)$. A more detailed analysis of $Y$ • and its counterpart for the $\bmod p$ Lie algebra $\mathfrak{g}=\operatorname{gr}(G) / \pi \operatorname{gr}(G)$ (we take $k=\mathbb{F}_{p}$ here) yields an isomorphism of graded $\mathbb{F}_{p}$-algebras

$$
H^{*}\left(G, \mathbb{F}_{p}\right) \stackrel{\sim}{\longrightarrow} H^{*}\left(\mathfrak{g}, \mathbb{F}_{p}\right) .
$$

Relating the cup products is a highly non-trivial step found in Laz65, Ch. V, Sec. 2.5.6]. Since $\mathfrak{g}$ is abelian its Lie algebra cohomology is $\Lambda \mathfrak{g}^{\vee}$, where $\mathfrak{g}^{\vee}=$ $\operatorname{Hom}_{\mathbb{F}_{p}}\left(\mathfrak{g}, \mathbb{F}_{p}\right) \simeq \operatorname{Hom}\left(G, \mathbb{F}_{p}\right)$. For the last identification note that homomorphisms $G \rightarrow \mathbb{F}_{p}$ factor through $G / G^{p}$ and as vector spaces $\operatorname{gr}(G)=\left(G / G^{p}\right) \oplus \pi \operatorname{gr}(G)$ when $(G, \omega)$ is saturated; cf. [Sch11, Lem. 26.10].

For an arbitrary finite coefficient field $k \supset \mathbb{F}_{p}$ we immediately deduce the more general result (via the Künneth formula for group cohomology, say), still assuming $(G, \omega)$ is equi- $p$-valued of course.

Corollary 8.3. $H^{*}(G, k) \stackrel{\sim}{\longrightarrow} \bigwedge \operatorname{Hom}(G, k) \simeq \bigwedge \mathfrak{g}^{\vee}$.

Here the exterior and the dual are taken over $k$.

There is a close connection between equi- $p$-valued groups and uniform pro- $p$ groups, as studied in DdSMS, Part I.4] for instance. The latter are topologically finitely generated pro- $p$-groups for which $G / \overline{G^{p}}$ is abelian (for $p>2$ ) and the lower $p$-series $G=G_{1} \geq G_{2} \geq \cdots$ has the property that the indices $\left[G_{i}: G_{i+1}\right]=p^{d}$ are independent of $i$. Recall that the lower $p$-series is defined as $G_{i+1}=G_{i}^{p}\left[G_{i}, G\right]$. One can verify that $\omega(g)=\sup \left\{i \in \mathbb{N}: g \in G_{i}\right\}$ defines a $p$-valuation for which $(G, \omega)$ becomes equi- $p$-valued with $t=1$. Conversely, Weigel's remarks summarized in [HKN11, Prop. A.1] show that a saturated equi- $p$-valued group is uniform.

\section{9. $A_{\infty}$-Algebras And their minimal models}

The notion of an $A_{\infty}$-algebra ("strongly homotopy associative algebra") first arose in Stasheff's 1961 Princeton Ph.D. thesis [Sta61. In topology loop spaces carry a natural operation, composition of loops, which is only associative up to homotopy. In homological algebra $A_{\infty}$-algebras naturally arise as follows. Suppose $A^{\bullet}$ is a complex of vector spaces, $B^{\bullet}$ is a DGA, and there is a quasi-isomorphism $i: A^{\bullet} \rightarrow B^{\bullet}$ along with a morphism of chain complexes $p: B^{\bullet} \rightarrow A^{\bullet}$ such that $i p \sim \operatorname{Id}_{B}$ ( "homotopy retract"). Say $\operatorname{Id}_{B} \bullet-i p=d h+h d$ for some homotopy $h: B^{\bullet} \rightarrow B^{\bullet}$. When one transfers the multiplication on $B^{\bullet}$ via $i$ and $p$ to $A^{\bullet}$ the resulting operation $m_{2}: A^{\bullet} \otimes A^{\bullet} \rightarrow A^{\bullet}$ turns out to only be associative up to homotopy, in the following sense: The "associator" $m_{2}\left(m_{2} \otimes 1\right)-m_{2}\left(1 \otimes m_{2}\right)$ lives 
in $\operatorname{Hom}\left(A^{\bullet \otimes 3}, A^{\bullet}\right)$, and one can explicitly write $m_{3}: A^{\bullet \otimes 3} \rightarrow A^{\bullet}$ in terms of $h$ for which

$$
m_{2}\left(m_{2} \otimes 1\right)-m_{2}\left(1 \otimes m_{2}\right)=\partial\left(m_{3}\right),
$$

where $\partial$ is the differential on the morphism complex. Continuing this way one finds a whole sequence of higher multiplications $m_{n}: A^{\bullet \otimes n} \rightarrow A^{\bullet}$ (of degree $2-n$ ) such that

$$
\sum_{r+s+t=n: 1<s<n}(-1)^{r s+t+1} m_{r+1+t}\left(1^{\otimes r} \otimes m_{s} \otimes 1^{\otimes t}\right)=\partial\left(m_{n}\right) .
$$

Note that $m_{1}=d_{A}$ • and for $n=2$ the above identity is just the Leibniz rule. This idea of homotopy transfer is explained and illustrated very clearly in [Val14, Ch. 1]. In the 1980's Kadeishvili developed the algebraic point of view further and laid the foundations in a series of papers $\operatorname{Kad} 83, \operatorname{Kad} 86, \operatorname{Kad} 88$, which unfortunately are hard to find (and in Russian). Some of his results were later re-proved by Merkulov in Mer99. Kontsevich made $A_{\infty}$-algebras a hot topic in his 1994 ICM paper Kon95, in which he proposed a connection to mirror symmetry via the Fukaya $A_{\infty}$-category of a symplectic manifold. The subject gained momentum and remains a very active research area today.

Keller has several excellent introductions to the subject, Kel01, Kel02, Kel06]. Below we will follow the conventions of Kel01.

Definition 9.1 ( Kel01, Def. 3.1]). An $A_{\infty}$-algebra over a field $k$ is a graded vector space $A^{\bullet}=\bigoplus_{i \in \mathbb{Z}} A^{i}$ endowed with homogeneous $k$-linear maps

$$
m_{n}: A^{\bullet \otimes n} \longrightarrow A^{\bullet} \quad n \geq 1,
$$

such that $m_{n}$ has degree $2-n$, and this sequence of higher multiplications $\left(m_{n}\right)_{n \geq 1}$ satisfies the following properties:

(1) $m_{1} \circ m_{1}=0$, so $\left(A^{\bullet}, m_{1}\right)$ is a complex.

(2) For $n \geq 1$ the Stasheff identities are satisfied. That is,

$$
\sum(-1)^{r+s t} m_{r+1+t}\left(1^{\otimes r} \otimes m_{s} \otimes 1^{\otimes t}\right)=0,
$$

where the sum runs over all decompositions $r+s+t=n$ with $s \geq 1$ (and $r, t$ non-negative).

(For $n=1$ the identity in (2) reduces to (1). For $n=2$ the identity shows that $m_{1}$ is a derivation.)

The Koszul sign convention is in force here when we apply $m_{n}$ to homogeneous elements (meaning when we swap $x$ and $y$ a $\operatorname{sign}(-1)^{\operatorname{deg}(x) \operatorname{deg}(y)}$ appears). For example, for homogeneous $a, b \in A^{\bullet}$ we have

$$
\left(m_{1} \otimes 1+1 \otimes m_{1}\right)(a \otimes b)=m_{1}(a) \otimes b+(-1)^{\operatorname{deg}(a)} a \otimes m_{1}(b)
$$

since we swap $m_{1}$ (of degree one) and $a$ in the second term.

Note that a DGA is the same thing as an $A_{\infty}$-algebra with $m_{n}=0$ for all $n \geq 3$. Furthermore the cohomology $h^{*}\left(A^{\bullet}\right)$ of any $A_{\infty}$-algebra $A^{\bullet}$ is a graded algebra under the multiplication induced by $m_{2}$. We emphasize that $h^{*}\left(A^{\bullet}\right)$ is an associative algebra although $A^{\bullet}$ is only associative up to homotopy. We often view $h^{*}\left(A^{\bullet}\right)$ as a minimal DGA (meaning it has differential $\left.d=0\right)$. One of the most fundamental results of the whole theory is the fact that $h^{*}\left(A^{\bullet}\right)$ carries an $A_{\infty^{-}}$ structure (unique up to non-canonical isomorphism). This is what we will discuss and utilize below.

First we recall how morphisms are defined. 
Definition 9.2 ([Kel01, Def. 3.4]). A morphism $f: A^{\bullet} \rightarrow B^{\bullet}$ between $A_{\infty}$-algebras $A^{\bullet}$ and $B^{\bullet}$ is a collection of homogeneous $k$-linear maps

$$
f_{n}: A^{\bullet \otimes n} \longrightarrow B^{\bullet} \quad n \geq 1,
$$

such that $f_{n}$ has degree $1-n$ and the sequence $\left(f_{n}\right)_{n \geq 1}$ satisfies the morphism identity $(\forall n)$

$$
\sum(-1)^{r+s t} f_{r+1+t}\left(1^{\otimes r} \otimes m_{s}^{A} \otimes 1^{\otimes t}\right)=\sum(-1)^{\sigma} m_{r}^{B}\left(f_{i_{1}} \otimes \cdots \otimes f_{i_{r}}\right) .
$$

Here the first sum runs over the same decompositions $r+s+t=n$ as in Definition $9.1(2)$, and the second sum runs over $1 \leq r \leq n$ and all decompositions $i_{1}+\cdots+i_{r}=$ $n$. The $\operatorname{sign}(-1)^{\sigma}$ is given by

$$
\sigma:=\sum_{j=1}^{r-1}(r-j)\left(i_{j}-1\right)=(r-1)\left(i_{1}-1\right)+(r-2)\left(i_{2}-1\right)+\cdots+\left(i_{r-1}-1\right) .
$$

(For $n=1$ this says that $f_{1}: A^{\bullet} \rightarrow B^{\bullet}$ is a morphism of complexes.) We say $f$ is a quasi-isomorphism if $f_{1}$ induces an isomorphism on cohomology $h^{*}\left(A^{\bullet}\right) \stackrel{\sim}{\longrightarrow} h^{*}\left(B^{\bullet}\right)$.

The identity morphism Id : $A^{\bullet} \rightarrow A^{\bullet}$ is the one with $\operatorname{Id}_{1}=\operatorname{Id}_{A} \bullet$ and $\operatorname{Id}_{n}=0$ for $n>1$, and there is a natural way to compose morphisms, which we will not recall here (cf. [Kel01, p. 10]), which yields the category of $A_{\infty}$-algebras over $k$.

Remark 9.3. In the applications we have in mind our $A_{\infty}$-algebras will be strictly unital, which means $A^{\bullet}$ contains a two-sided multiplicative identity $1=1_{A} \bullet \in A^{0}$ for $m_{2}$ with the property $m_{n}\left(a_{1} \otimes \cdots \otimes a_{n}\right)=0$ if $n \neq 2$ and some $a_{i}=1$. A morphism $f: A^{\bullet} \rightarrow B^{\bullet}$ between strictly unital $A_{\infty}$-algebras is said to be strictly unital if $f_{1}\left(1_{A} \bullet\right)=1_{B} \bullet$ and $f_{n}\left(a_{1} \otimes \cdots \otimes a_{n}\right)=0$ if $n>1$ and some $a_{i}=1$.

The following key result of Kadeishvili is usually referred to as the minimal model theorem.

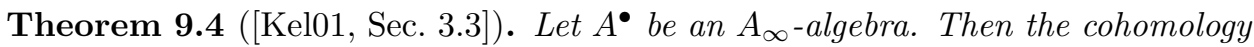
$h^{*}\left(A^{\bullet}\right)$ has an $A_{\infty}$-structure $\left(\mu_{n}\right)_{n \geq 1}$ with the following properties.

(1) $\mu_{1}=0$ ("minimality") and $\mu_{2}$ is induced by the $m_{2}$ on $A^{\bullet}$.

(2) There is a quasi-isomorphism of $A_{\infty}$-algebras,

$$
f: h^{*}\left(A^{\bullet}\right) \longrightarrow A^{\bullet},
$$

lifting the identity on $h^{*}\left(A^{\bullet}\right)$; that is, $h^{i}\left(f_{1}\right)=\operatorname{Id}_{h^{i}\left(A^{\bullet}\right)}$ for all $i \in \mathbb{Z}$.

This $A_{\infty}$-structure $\left(\mu_{n}\right)_{n \geq 1}$ is uniquely determined by (1) and (2) up to (noncanonical) isomorphisms of $A_{\infty}$-algebras.

Eventually we will apply this result to the DGA $\mathcal{H}^{\bullet}$ and get an $A_{\infty}$-structure on $h^{*}\left(\mathcal{H}^{\bullet}\right) \simeq E(\Omega)$.

The simple idea behind Theorem 9.4 is very similar to the homotopy transfer discussed in the first paragraph of this section: Say we start with an $A_{\infty}$-algebra $A^{\bullet}$, or even just a DGA. For each $i$ let $B^{i} \subset Z^{i}$ be the coboundaries and cocycles in $A^{i}$ and once and for all choose vector space complements

$$
Z^{i}=B^{i} \oplus H^{i} \quad A^{i}=Z^{i} \oplus C^{i} \quad\left(=B^{i} \oplus H^{i} \oplus C^{i}\right) .
$$

Let $i: H^{\bullet} \rightarrow A^{\bullet}$ be the inclusion map and let $p: A^{\bullet} \rightarrow H^{\bullet}$ be the projection map. Clearly $p i=\operatorname{Id}_{H}$. On the other hand $i p \sim \operatorname{Id}_{A} \bullet$. To build a homotopy 
$h: A^{\bullet} \rightarrow A^{\bullet}$ such that $\operatorname{Id}_{A} \bullet-i p=d h+h d$ first observe that the composition

$$
C^{i-1} \longrightarrow A^{i-1} \stackrel{d}{\longrightarrow} B^{i}
$$

is an isomorphism. This is immediate. Let $\gamma^{i}: B^{i} \rightarrow C^{i-1}$ denote its inverse, and extend it to a map $h^{i}: A^{i} \rightarrow C^{i-1}$ by setting $h^{i}=0$ on $H^{i} \oplus C^{i}$. Unwinding these definitions it is easily checked that $d \circ h^{i}$ is the projection $A^{i} \rightarrow B^{i}$ and that $h^{i+1} \circ d$ is the projection $A^{i} \rightarrow C^{i}$. Thus indeed $\operatorname{Id}_{A} \bullet-i p=d h+h d$. Now one simply transfers the $A_{\infty}$-structure on $A^{\bullet}$ to $H^{\bullet} \simeq h^{*}\left(A^{\bullet}\right)$ via $i$ and $p$. One can explicitly write the higher multiplications $\left(\mu_{n}\right)_{n \geq 1}$ in terms of the homotopy $h$ and $\left(m_{n}\right)_{n \geq 1}$; cf. [LPWZb, Thm. 2.2] and [WE18, Ex. 5.2.8] for DGA's. Same for the quasi-isomorphism $H^{\bullet} \rightarrow A^{\bullet}$; cf. [LPWZb, Prop. 2.3].

\section{0. $A_{\infty}$-Modules And THE Derived CATEGory}

Let $A^{\bullet}$ be an $A_{\infty}$-algebra. A left $A_{\infty}$-module over $A^{\bullet}$ is a graded $k$-vector space $M^{\bullet}=\bigoplus_{i \in \mathbb{Z}} M^{i}$ with homogeneous $k$-linear maps

$$
\nu_{n}: A^{\bullet \otimes n-1} \otimes M^{\bullet} \longrightarrow M^{\bullet} n \geq 1,
$$

where $\nu_{n}$ has degree $2-n$ and which satisfy the analogues of the Stasheff identities (Definition 9.1(2)):

$$
\sum(-1)^{r+s t} \nu_{r+1+t}\left(1^{\otimes r} \otimes m_{s} \otimes 1^{\otimes t}\right)=0 .
$$

(When $t=0$ the term $\nu_{r+1+t}\left(1^{\otimes r} \otimes m_{s} \otimes 1^{\otimes t}\right)$ is interpreted as $\nu_{r+1}\left(1^{\otimes r} \otimes \nu_{s}\right)$.) For $n=1$ this says that $\nu_{1} \circ \nu_{1}=0$. An $A_{\infty}$-module structure on a complex $\left(M^{\bullet}, \nu_{1}\right)$ amounts to a morphism of $A_{\infty}$-algebras $A^{\bullet} \rightarrow \operatorname{End}_{k}^{\bullet}\left(M^{\bullet}\right)$ where the target is thought of as a DGA. In all our applications $A^{\bullet}$ will be strictly unital; cf. Remark 9.3. We will henceforth exclusively consider strictly unital $A_{\infty}$-modules $M^{\bullet}$, which means $\nu_{2}(1 \otimes x)=x$ for all $x \in M^{\bullet}$ and $\nu_{n}\left(a_{2} \otimes \cdots \otimes a_{n} \otimes x\right)=0$ for $n>2$ if some $a_{i}=1$.

Morphisms between $A_{\infty}$-modules $M^{\bullet}$ and $N^{\bullet}$ are defined analogously to Definition 9.2 A morphism $f: M^{\bullet} \rightarrow N^{\bullet}$ is a collection of homogeneous $k$-linear maps,

$$
f_{n}: A^{\bullet \otimes n-1} \otimes M^{\bullet} \longrightarrow N^{\bullet} \quad n \geq 1,
$$

where $f_{n}$ has degree $1-n$, which are required to satisfy the identity (cf. Kel01, eqn. (4.2), p. 15])

$$
\sum(-1)^{r+s t} f_{r+1+t}\left(1^{\otimes r} \otimes m_{s} \otimes 1^{\otimes t}\right)=\sum(-1)^{u(v+1)} \nu_{u+1}\left(1^{\otimes u} \otimes f_{v}\right)
$$

with conventions in the $t=0$ case as above. On the right we are summing over decompositions $n=u+v$ with $v \geq 1$ and $u$ non-negative. Again we will only consider strictly unital $f$, which means $f_{n}\left(a_{2} \otimes \cdots \otimes a_{n} \otimes x\right)=0$ for $n>1$ if some $a_{i}=1$.

There is an obvious identity morphism Id : $M^{\bullet} \rightarrow M^{\bullet}$ and a natural way to compose morphisms, which results in the category $C_{\infty}\left(A^{\bullet}\right)$ of strictly unital left $A_{\infty}$-modules over $A^{\bullet}$, with strictly unital morphisms.

There is a notion of a morphism $f: M^{\bullet} \rightarrow N^{\bullet}$ being null-homotopic, which we will not recall in detail here; see [Kel01, p. 16] for the explicit formula expressing $f_{n}$ in terms of a homotopy $h_{n}: A^{\bullet \otimes n-1} \otimes M^{\bullet} \rightarrow N^{\bullet}$ (here $h_{n}$ has degree $-n$ ). In the homotopy category $K_{\infty}\left(A^{\bullet}\right)$ morphisms are taken modulo homotopy. To arrive 
at the derived category we should invert all quasi-isomorphisms in $K_{\infty}\left(A^{\bullet}\right)$, but another fundamental result of Kadeishvili says this is unnecessary:

Theorem 10.1 ([Kel01, Thm. 4.2]). Let $f: M^{\bullet} \rightarrow N^{\bullet}$ be a quasi-isomorphism of $A_{\infty}$-modules. Then $f$ has a homotopy inverse morphism $g: N^{\bullet} \rightarrow M^{\bullet}$ of $A_{\infty}$ modules $\left(f \circ g \sim \operatorname{Id}_{N} \bullet\right.$ and $\left.g \circ f \sim \operatorname{Id}_{M} \bullet\right)$.

Thus $D_{\infty}\left(A^{\bullet}\right)=K_{\infty}\left(A^{\bullet}\right)$ is the derived category of $A_{\infty}$-modules over $A^{\bullet}$. It naturally becomes a triangulated category as discussed in [Kel01, Prop. 5.2]. For instance the translation functor is given by the shifts $T\left(M^{\bullet}\right)^{i}=M^{i+1}$ with higher multiplications $m_{n}^{T\left(M^{\bullet}\right)}=(-1)^{n} m_{n}^{M^{\bullet}}$.

When $A^{\bullet}$ is a DGA any DG-module is of course an $A_{\infty}$-module (with $\nu_{n}=0$ for $n \geq 3$ ), but not conversely. Given a complex $\left(M^{\bullet}, \nu_{1}\right)$ there could be morphisms $A^{\bullet} \rightarrow \operatorname{End}_{k}^{\bullet}\left(M^{\bullet}\right)$ of $A_{\infty}$-algebras which do not arise from a DG-module (cf. the last paragraph of Kel01, p. 15]). However, on the level of derived categories we have an equivalence by a result from Lefèvre-Hasegawa's 2003 Paris 7 Ph.D. thesis.

Lemma 10.2 ([LH03, Lem. 4.1.3.8]). Let $A^{\bullet}$ be a DGA (unital by our conventions). The inclusion of the category of (unital) DG-modules $C\left(A^{\bullet}\right) \rightarrow C_{\infty}\left(A^{\bullet}\right)$ induces an equivalence of triangulated categories

$$
D\left(A^{\bullet}\right) \stackrel{\sim}{\longrightarrow} D_{\infty}\left(A^{\bullet}\right) .
$$

Another key result from Lefèvre-Hasegawa's treatise which we will need concerns restriction of modules along a morphism of $A_{\infty}$-algebras, $f: A^{\bullet} \rightarrow B^{\bullet}$. Say $M^{\bullet}$ is an $A_{\infty}$-module over $B^{\bullet}$ with higher structure maps $\left(\nu_{n}^{B}\right)$. On the same vector space $M^{\bullet}$ we define an $A_{\infty}$-module structure over $A^{\bullet}$ by letting

$$
\nu_{n}^{A}: A^{\bullet \otimes n-1} \otimes M^{\bullet} \longrightarrow M^{\bullet} \quad \nu_{n}^{A}=\sum(-1)^{\sigma} \nu_{r+1}^{B}\left(f_{i_{1}} \otimes \cdots \otimes f_{i_{r}} \otimes \mathrm{Id}\right),
$$

where the sum extends over $1 \leq r \leq n-1$ and decompositions $i_{1}+\cdots+i_{r}=n-1$. The sign $(-1)^{\sigma}$ is as in Definition 9.2 cf. [Kel01, Sec. 6.2]. The ensuing module is denoted by $f^{*} M^{\bullet}$.

Theorem 10.3 ([LH03, Thm. 4.1.2.4]). If $f: A^{\bullet} \rightarrow B^{\bullet}$ is a quasi-isomorphism of $A_{\infty}$-algebras, the restriction functor $f^{*}: C_{\infty}\left(B^{\bullet}\right) \rightarrow C_{\infty}\left(A^{\bullet}\right)$ induces an equivalence of triangulated categories

$$
f^{*}: D_{\infty}\left(B^{\bullet}\right) \stackrel{\sim}{\longrightarrow} D_{\infty}\left(A^{\bullet}\right) .
$$

In our applications $A^{\bullet}$ will be a DGA, and we endow $h^{*}\left(A^{\bullet}\right)$ with an $A_{\infty}$ structure as in Kadeishvili's Theorem [9.4. By Lemma 10.2 and Theorem 10.3 a choice of quasi-isomorphism $f: h^{*}\left(A^{\bullet}\right) \rightarrow A^{\bullet}$ gives an equivalence $f^{*}: D\left(A^{\bullet}\right) \stackrel{\sim}{\longrightarrow}$ $D_{\infty}\left(h^{*}\left(A^{\bullet}\right)\right)$ of triangulated categories.

\section{Putting the Pieces together}

11.1. Proof of Theorem 1.1. We return to the setup of section 6. Thus $k$ is a finite field of characteristic $p>0$ and $G$ is a compact $p$-adic Lie group which is assumed to be torsionfree and pro- $p$. Recall that $\Omega=k \llbracket G \rrbracket$ and $D(\Omega)$ is the derived category of pseudocompact $\Omega$-modules. We fix a projective resolution $P^{\bullet} \rightarrow k$ and consider the DGA $\mathcal{H}^{\bullet} \stackrel{\sim}{\longrightarrow} \operatorname{End}_{\Omega}^{\bullet}\left(P^{\bullet}\right)$ whose cohomology is the Yoneda algebra $h^{*}\left(\mathcal{H}^{\bullet}\right) \stackrel{\sim}{\longrightarrow} \operatorname{Ext}_{\Omega}^{*}(k, k)=E(\Omega)$. Furthermore, by Schneider's Theorem [5.6 and Pontryagin duality (Corollary 3.2) there is an equivalence

$$
D(\Omega)^{\mathrm{op}} \stackrel{\sim}{\longrightarrow} D\left(\operatorname{End}_{\Omega}^{\bullet}\left(P^{\bullet}\right)\right)
$$


which on objects takes a $K$-projective complex $X^{\bullet}$ of pseudocompact $\Omega$-modules to the left DG module $\operatorname{Hom}_{\Omega}^{\bullet}\left(X^{\bullet}, P^{\bullet}\right)$. Now, by Kadeishvili's Theorem 9.4 the cohomology $h^{*}\left(\mathcal{H}^{\bullet}\right) \stackrel{\sim}{\longrightarrow} E(\Omega)$ acquires a natural $A_{\infty}$-algebra structure (unique up to non-unique isomorphism), and we may choose a quasi-isomorphism of $A_{\infty}$ algebras $f: h^{*}\left(\mathcal{H}^{\bullet}\right) \rightarrow \mathcal{H}^{\bullet}$ which by Lemma 10.2 and Theorem 10.3 yields an equivalence

$$
f^{*}: D\left(\mathcal{H}^{\bullet}\right) \stackrel{\sim}{\longrightarrow} D_{\infty}(E(\Omega)) .
$$

Altogether this gives an equivalence $D(\Omega)^{\text {op }} \stackrel{\sim}{\longrightarrow} D_{\infty}(E(\Omega))$ taking $X^{\bullet}$ to $f^{*} \operatorname{Hom}_{\Omega}^{\bullet}\left(X^{\bullet}, P^{\bullet}\right)$.

Remark 11.1. There is a natural way to define the opposite $A^{\bullet \text { op }}$ of an $A_{\infty}$ algebra $A^{\bullet}$; cf. [LPWZa, Sec. 3.5]. We take the same graded vector space, but the opposite higher multiplication maps $m_{n}^{\text {op }}:\left(A^{\bullet \circ p}\right)^{\otimes n} \rightarrow A^{\bullet \text { op }}$ are defined as $m_{n}^{\text {op }}=(-1)^{\left(\begin{array}{l}n \\ 2\end{array}\right)+1} \cdot m_{n} \circ$ (reverse), where "reverse" is the map reversing the factors in the tensor product. For example, when applied to homogeneous elements $a_{i}$ we have

$$
m_{n}^{\mathrm{op}}\left(a_{1} \otimes \cdots \otimes a_{n}\right)=(-1)^{\left(\begin{array}{l}
n \\
2
\end{array}\right)+1+\sum_{i<j} \operatorname{deg}\left(a_{i}\right) \operatorname{deg}\left(a_{j}\right)} \cdot m_{n}\left(a_{n} \otimes \cdots \otimes a_{1}\right) .
$$

By [LPWZa, Lem. 3.3] this gives an $A_{\infty}$-algebra $A^{\bullet \text { op }}$, and one can easily verify that there is an equivalence

$$
D_{\infty}\left(A^{\bullet \text { op }}\right) \stackrel{\sim}{\longrightarrow} D_{\infty}\left(A^{\bullet}\right)^{\text {op }} .
$$

(The opposite of a triangulated category was defined in section 2])

We define the Koszul dual of $\Omega$ as follows.

Definition 11.2. $\Omega^{!}=E(\Omega)^{\mathrm{op}}=\operatorname{Ext}_{\Omega}^{*}(k, k)^{\mathrm{op}}$ (with its $A_{\infty}$-structure).

Then we conclude that $D(\Omega) \stackrel{\sim}{\longrightarrow} D_{\infty}\left(\Omega^{!}\right)$by taking opposites on both sides above.

11.2. Proof of Theorem 1.2. Now let $G$ be a uniform pro- $p$ group. As explained after Corollary 8.3 it carries a natural $p$-valuation $\omega$ such that $(G, \omega)$ becomes equi- $p$-valued with $t=1$ and saturated. Thus

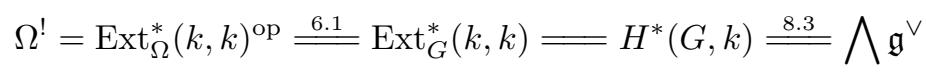

as graded $k$-algebras from which the first half of Theorem 1.2 follows.

It remains to show that $\mu_{n}=0$ for $n>2$ when $G$ is abelian. Note that any abelian $p$-valuable group $G$ of rank $d$ is topologically isomorphic to $\mathbb{Z}_{p}^{d}$ by choosing a basis $\left(g_{1}, \ldots, g_{d}\right)$. In particular we can always endow $G$ with a $p$-valuation $\omega$ in such a way that $(G, \omega)$ becomes equi- $p$-valued with $t=1$ and saturated: just let $\omega(g)=1+\min _{i=1, \ldots, d} v\left(x_{i}\right)$ when $g=g_{1}^{x_{1}} \cdots g_{d}^{x_{d}}$. As always we are assuming $p>2$ here. Correspondingly we have an isomorphism of topological filtered $k$-algebras,

$$
k \llbracket X_{1}, \ldots, X_{d} \rrbracket \stackrel{\sim}{\longrightarrow} \Omega \quad \sum_{\alpha} c_{\alpha} X^{\alpha} \mapsto \sum_{\alpha} c_{\alpha} \mathbf{b}^{\alpha} .
$$

We prefer to free $\Omega$ from coordinates and think of it as a completed symmetric algebra as follows. Fix a $d$-dimensional vector space $V$ over $k$ and consider the $I$-adic completion $\widehat{S(V)}=\lim S(V) / I^{i}$ where $I=\operatorname{ker}(S(V) \longrightarrow k)$ is the augmentation ideal. Fixing a basis $\left.\overleftarrow{\left(e_{1}\right.}, \ldots, e_{d}\right)$ for $V$ the map which takes $\mathbf{e}^{\alpha}=e_{1}^{\alpha_{1}} \cdots e_{d}^{\alpha_{d}}$ to $\mathbf{b}^{\alpha}$ extends to an isomorphism $\widehat{S(V)} \stackrel{\sim}{\longrightarrow} \Omega$ of topological $k$-algebras. Note that $\widehat{S(V)}$ 
is flat over $S(V)$ so $I$-adic completion is exact on the category of finitely generated $S(V)$-modules. In particular we obtain a projective resolution $P^{\bullet} \rightarrow k$ in $\operatorname{Mod}(\Omega)$ by taking the $I$-adic completion of the Koszul resolution $K^{\bullet}$. That is,

$$
K^{\bullet} \cdots \longrightarrow S(V) \otimes \bigwedge^{2} V \longrightarrow S(V) \otimes \bigwedge^{1} V \longrightarrow S(V) \longrightarrow k \longrightarrow 0
$$

and

$$
P^{\bullet} \cdots \longrightarrow \widehat{S(V)} \otimes \bigwedge^{2} V \longrightarrow \widehat{S(V)} \otimes \bigwedge^{1} V \longrightarrow \widehat{S(V)} \longrightarrow k \longrightarrow 0 .
$$

Recall that we use cohomological indexing, which means $K^{-i}=S(V) \otimes \bigwedge^{i} V$ and similarly for $P^{\bullet}$. We use this particular completed Koszul resolution $P^{\bullet}$ in the definition of $\mathcal{H}^{\bullet}$. That is, $\mathcal{H}^{\bullet}=\operatorname{End}_{\Omega}^{\bullet}\left(P^{\bullet}\right)$, which has cohomology $h^{*}\left(\mathcal{H}^{\bullet}\right)=E(\Omega)$. By Theorem 9.4 the $A_{\infty}$-structure on $E(\Omega)$ is characterized up to $A_{\infty}$-isomorphism by the fact that there is a quasi-isomorphism

$$
f: h^{*}\left(\mathcal{H}^{\bullet}\right)=E(\Omega) \longrightarrow \mathcal{H}^{\bullet} .
$$

Analogously we have the differential graded algebra $\operatorname{End}_{S(V)}^{\bullet}\left(K^{\bullet}\right)$ obtained from the (actual) Koszul resolution, which has cohomology $E(S(V))$. Again by Theorem 9.4 there is an $A_{\infty}$-structure on $E(S(V))$ admitting a quasi-isomorphism

$$
g: h^{*}\left(\operatorname{End}_{S(V)}^{\bullet}\left(K^{\bullet}\right)\right)=E(S(V)) \longrightarrow \operatorname{End}_{S(V)}^{\bullet}\left(K^{\bullet}\right) .
$$

By a well-known result a finitely generated graded algebra $A$ is Koszul if and only if the $A_{\infty}$-algebra $\operatorname{Ext}_{A}^{*}(k, k)$ is formal; cf. Kel02, Sec. 2.2] and Wit18, Thm. 6.3.3]. We deduce that $E(S(V))$ has the trivial $A_{\infty}$-structure since $S(V)$ is Koszul. We are therefore done once we check that the natural map

$$
\operatorname{End}_{S(V)}^{\bullet}\left(K^{\bullet}\right) \longrightarrow \operatorname{End}_{\widehat{S(V)}}^{\bullet}\left(P^{\bullet}\right)
$$

which takes a homogeneous $a=\left(a_{q}\right)_{q \in \mathbb{Z}}$ to the corresponding sequence $\hat{a}=\left(\hat{a}_{q}\right)_{q \in \mathbb{Z}}$ of maps between completions, is a quasi-isomorphism. This follows from the standard calculation (and the trivial observation that the differentials of $\operatorname{Hom}_{S(V)}\left(S(V) \otimes \bigwedge^{\bullet} V, k\right)$ all vanish since $V \hookrightarrow S(V)$ augments to zero)

$$
\begin{aligned}
& h^{i}\left(\operatorname{End}_{S(V)}^{\bullet}\left(K^{\bullet}\right)\right)=\operatorname{Ext}_{S(V)}^{i}(k, k) \\
& =h^{i}\left(\operatorname{Hom}_{S(V)}\left(K^{\bullet}, k\right)\right) \\
& =h^{i}\left(\operatorname{Hom}_{S(V)}(S(V) \otimes \grave{\bigwedge} V, k)\right) \\
& =h^{i}\left(\operatorname{Hom}_{k}(\grave{\bigwedge} V, k)\right) \\
& =\bigwedge^{i} V^{\vee}
\end{aligned}
$$

together with the completely analogous computation of the cohomology of End $\overline{\widehat{S(V)}}\left(P^{\bullet}\right)$, which in particular shows that, as graded $k$-algebras, we have isomorphisms

$$
E(S(V)) \stackrel{\sim}{\longrightarrow} E(\widehat{S(V)}) \stackrel{\sim}{\longrightarrow} \bigwedge V^{\vee}
$$

Since they are all minimal models of $\mathcal{H}^{\bullet}$ they are $A_{\infty}$-isomorphic. As observed above $E(S(V))$ has a trivial $A_{\infty}$-structure, and consequently so does $E(\Omega)$ and its opposite $\Omega^{!}=\bigwedge \mathfrak{g}^{\vee}$. This finishes the proof. 
Remark 11.4. The case $d=1$ of Theorem 1.2 is due to Schneider. In Sch15, Sec. 5.2(4)] it is shown that there is an equivalence $D\left(\mathbb{Z}_{p}\right) \stackrel{\sim}{\longrightarrow} D(k[\varepsilon])$ where $k[\varepsilon]=$ $k \bigoplus k \varepsilon$ is the algebra of dual numbers $\left(\varepsilon^{2}=0\right)$ thought of as a DGA concentrated in degrees 0 and 1 , with zero differential. Note that DG-modules over $k[\varepsilon]$ are the same as graded $k$-vector spaces $M^{\bullet}=\bigoplus_{i \in \mathbb{Z}} M^{i}$ with two anti-commuting differentials $d$ and $\varepsilon$ of degree one, i.e., $d \varepsilon+\varepsilon d=0$. Obviously $k[\varepsilon] \simeq \bigwedge k$, so Schneider's result also follows from Theorem 1.2 by observing that $\Lambda k$ is intrinsically formal (every minimal $A_{\infty}$-structure on it is trivial), which can be deduced from a computation of its Hochschild cohomology $H H^{*}(\bigwedge k, \bigwedge k)$ as in [Wit18, Ex. 1.1.16] together with Kadeishvili's criterion [Kad09, Thm. 6.3], which says that every $A_{\infty}$-structure on a graded algebra $R$ is degenerate if $H H^{n, 2-n}(R, R)=0$ for all $n>2$.

Note that when $G$ is abelian the DGA $\wedge \mathfrak{g}^{\vee}$ is graded commutative and therefore isomorphic to its opposite $\left(\Lambda \mathfrak{g}^{\vee}\right)^{\mathrm{op}}$. (We refer the reader to LPWZa, Sec. 3.4] for its definition.) As a result Theorem 1.2 may also be thought of as an equivalence $D(G) \stackrel{\sim}{\longrightarrow} D\left(\bigwedge \mathfrak{g}^{\vee}\right)$ in the case where $G \simeq \mathbb{Z}_{p}^{d}$. Once we fix a basis $\left(\xi_{i}\right)$ for $\mathfrak{g}$, a DGmodule over $\Lambda \mathfrak{g}^{\vee}$ can be identified with a graded $k$-vector space $M^{\bullet}=\bigoplus_{i \in \mathbb{Z}} M^{i}$ with $d+1$ anti-commuting degree one differentials $d, \varepsilon_{1}, \ldots, \varepsilon_{d}$. We apologize for the double meaning of $d$, which refers to both $d_{M} \bullet$ and $\operatorname{dim} G$. (Here $\varepsilon_{i}$ is the action of the dual basis vector $\xi_{i}^{\vee} \in \mathfrak{g}^{\vee}$ on $M^{\bullet}$.)

\section{Open Ends AND UnANSWERED QUESTIONS}

We finish with a few questions which have not been addressed in this paper.

(a) By Theorem 1.1 one can recover $\Omega=k \llbracket G \rrbracket$ up to derived equivalence from the $A_{\infty}$-algebra $\Omega^{!}=\operatorname{Ext}_{\Omega}^{*}(k, k)^{\mathrm{op}}$. Does $\Omega^{!}$determine $\Omega$ up to isomorphism?

(b) Is there a converse to Theorem 1.2 in the sense that $G$ must be abelian if the $A_{\infty}$-structure on $\bigwedge \mathfrak{g}^{\vee}$ is trivial?

(c) Suppose $H \subset G$ is an open subgroup. Then $\Omega(G)$ is finite free over the subalgebra $\Omega(H)$, and we have the restriction $\operatorname{map} \operatorname{Mod} \Omega(G) \rightarrow \operatorname{Mod} \Omega(H)$ which induces a map $D(\Omega(G)) \rightarrow D(\Omega(H))$. Is there a morphism of $A_{\infty^{-}}$ algebras $\bigwedge \mathfrak{g}^{\vee} \longrightarrow \wedge \mathfrak{h}^{\vee}$ inducing the corresponding map on $D_{\infty}$ via "extension of scalars" along this map?

Several experts in the field have independently confirmed that the answer to all three questions is yes, but it would take us too far afield to try to reproduce their arguments here.

In fact there is a recent preprint WE19 by Carl Wang-Erickson in which he answers these questions affirmatively as an application of the techniques he developed in WE18. The latter provide a natural framework to deal with these problems. Indeed WE19, Cor. 3.1.6] gives a completely explicit presentation for $\Omega$ in terms of $\Omega$ !

\section{ACKNOWLEDGMENTS}

This work emerged from several inspiring conversations with Peter Schneider during my visit to Münster in July 2015 and after. He led me to think about Koszul duality and $A_{\infty}$-algebras, and I am truly grateful for his guidance. The inspiration drawn from Sch15 should be clear to the reader. After sending out an initial draft of the article in the summer of 2018, I received tremendously helpful remarks 
and suggestions from Carl Wang-Erickson, Michael Harris, Bernhard Keller, Karol Koziol, Leonid Positselski, Niccolò Ronchetti, and Peter Schneider. Special thanks go to Positselski for making extensive comments and sharing his expertise in the area, as well as Harris for pointing to potential applications.

\section{REFERENCES}

[BGG78] I. N. Bernšteĭn, I. M. Gel'fand, and S. I. Gel'fand, Algebraic vector bundles on $\mathbf{P}^{n}$ and problems of linear algebra (Russian), Funktsional. Anal. i Prilozhen. 12 (1978), no. 3, 66-67. MR.509387

[BGS96] Alexander Beilinson, Victor Ginzburg, and Wolfgang Soergel, Koszul duality patterns in representation theory, J. Amer. Math. Soc. 9 (1996), no. 2, 473-527, DOI 10.1090/S0894-0347-96-00192-0. MR 1322847

[BL94] Joseph Bernstein and Valery Lunts, Equivariant sheaves and functors, Lecture Notes in Mathematics, vol. 1578, Springer-Verlag, Berlin, 1994. MR.1299527

[Bru66] Armand Brumer, Pseudocompact algebras, profinite groups and class formations, J. Algebra 4 (1966), 442-470, DOI 10.1016/0021-8693(66)90034-2. MR202790

[CE99] Henri Cartan and Samuel Eilenberg, Homological algebra, with an appendix by David A. Buchsbaum, reprint of the 1956 original, Princeton Landmarks in Mathematics, Princeton University Press, Princeton, NJ, 1999. MR.1731415

[DdSMS] J. D. Dixon, M. P. F. du Sautoy, A. Mann, and D. Segal, Analytic pro-p groups, 2nd ed., Cambridge Studies in Advanced Mathematics, vol. 61, Cambridge University Press, Cambridge, 1999. MR 1720368

[Dem86] Michel Demazure, Lectures on p-divisible groups, reprint of the 1972 original, Lecture Notes in Mathematics, vol. 302, Springer-Verlag, Berlin, 1986. MR883960

[Eme10] Matthew Emerton, Ordinary parts of admissible representations of $p$-adic reductive groups I. Definition and first properties (English, with English and French summaries), Astérisque 331 (2010), 355-402. MR2667882

[Flo06] Gunnar Fløystad, Koszul duality and equivalences of categories, Trans. Amer. Math. Soc. 358 (2006), no. 6, 2373-2398, DOI 10.1090/S0002-9947-05-04035-3. MR2204036

[Gab62] Pierre Gabriel, Des catégories abéliennes (French), Bull. Soc. Math. France 90 (1962), 323-448. MR:232821

[Har66] Robin Hartshorne, Residues and duality, Lecture notes of a seminar on the work of A. Grothendieck, given at Harvard 1963/64, with an appendix by P. Deligne, Lecture Notes in Mathematics, No. 20, Springer-Verlag, Berlin-New York, 1966. MR0222093

[HKN11] Annette Huber, Guido Kings, and Niko Naumann, Some complements to the Lazard isomorphism, Compos. Math. 147 (2011), no. 1, 235-262, DOI 10.1112/S0010437X10004884. MR2771131

[Kad83] T. V. Kadeishvili, The algebraic structure in the homology of an $A(\infty)$-algebra (Russian, with English and Georgian summaries), Soobshch. Akad. Nauk Gruzin. SSR 108 (1982), no. 2, 249-252 (1983). MR720689

[Kad86] T. V. Kadeishvili, Twisted tensor products for the category of $A(\infty)$-algebras and $A(\infty)$ modules (Russian, with English summary), Trudy Tbiliss. Mat. Inst. Razmadze Akad. Nauk Gruzin. SSR 83 (1986), 26-45. MR881043

[Kad88] T. V. Kadeishvili, The structure of the $A(\infty)$-algebra, and the Hochschild and Harrison cohomologies (Russian, with English summary), Trudy Tbiliss. Mat. Inst. Razmadze Akad. Nauk Gruzin. SSR 91 (1988), 19-27. MR1029003

[Kad09] Tornike Kadeishvili, Cohomology $C_{\infty}$-algebra and rational homotopy type, Algebraic topology — old and new, Banach Center Publ., vol. 85, Polish Acad. Sci. Inst. Math., Warsaw, 2009, pp. 225-240, DOI 10.4064/bc85-0-16. MR2503530

[Kel94] Bernhard Keller, Deriving DG categories, Ann. Sci. École Norm. Sup. (4) 27 (1994), no. 1, 63-102. MR.1258406

[Kel01] Bernhard Keller, Introduction to A-infinity algebras and modules, Homology Homotopy Appl. 3 (2001), no. 1, 1-35, DOI 10.4310/hha.2001.v3.n1.a1. MR1854636

[Kel02] Bernhard Keller, A-infinity algebras in representation theory, Representations of algebra. Vol. I, II, Beijing Norm. Univ. Press, Beijing, 2002, pp. 74-86. MR2067371 
[Kel06] Bernhard Keller, A-infinity algebras, modules and functor categories, Trends in representation theory of algebras and related topics, Contemp. Math., vol. 406, Amer. Math. Soc., Providence, RI, 2006, pp. 67-93, DOI 10.1090/conm/406/07654. MR2258042

[Kel07] Bernhard Keller, Derived categories and tilting, Handbook of tilting theory, London Math. Soc. Lecture Note Ser., vol. 332, Cambridge Univ. Press, Cambridge, 2007, pp. 49-104, DOI 10.1017/CBO9780511735134.005. MR.2384608

[Koh17] Jan Kohlhaase, Smooth duality in natural characteristic, Adv. Math. 317 (2017), 1-49, DOI 10.1016/j.aim.2017.06.038. MR.3682662

[Kon95] Maxim Kontsevich, Homological algebra of mirror symmetry, Proceedings of the International Congress of Mathematicians, Vol. 1, 2 (Zürich, 1994), Birkhäuser, Basel, 1995, pp. 120-139. MR.1403918

[Kra07] Henning Krause, Derived categories, resolutions, and Brown representability, Interactions between homotopy theory and algebra, Contemp. Math., vol. 436, Amer. Math. Soc., Providence, RI, 2007, pp. 101-139, DOI 10.1090/conm/436/08405. MR 2355771

[Laz65] Michel Lazard, Groupes analytiques p-adiques (French), Inst. Hautes Études Sci. Publ. Math. 26 (1965), 389-603. MR209286

[LH03] K. Lefèvre-Hasegawa, Sur les $A_{\infty}$-catégories, Thèse de Doctorat, Universite Paris 7 , November 2003. Available online at arXiv:math/0310337

[LPWZa] Di Ming Lu, John H. Palmieri, Quan Shui Wu, and James J. Zhang, Koszul equivalences in $A_{\infty}$-algebras, New York J. Math. 14 (2008), 325-378. MR2430869

[LPWZb] D.-M. Lu, J. H. Palmieri, Q.-S. Wu, and J. J. Zhang, A-infinity structure on Ext-algebras, J. Pure Appl. Algebra 213 (2009), no. 11, 2017-2037, DOI 10.1016/j.jpaa.2009.02.006. MR 2533303

[Mer99] S. A. Merkulov, Strong homotopy algebras of a Kähler manifold, Internat. Math. Res. Notices 3 (1999), 153-164, DOI 10.1155/S1073792899000070. MR.1672242

[MOS09] Volodymyr Mazorchuk, Serge Ovsienko, and Catharina Stroppel, Quadratic duals, Koszul dual functors, and applications, Trans. Amer. Math. Soc. 361 (2009), no. 3, 1129-1172, DOI 10.1090/S0002-9947-08-04539-X. MR2457393

[Nee01] Amnon Neeman, Triangulated categories, Annals of Mathematics Studies, vol. 148, Princeton University Press, Princeton, NJ, 2001. MR.1812507

[OS18] Rachel Ollivier and Peter Schneider, A canonical torsion theory for pro-p Iwahori-Hecke modules, Adv. Math. 327 (2018), 52-127, DOI 10.1016/j.aim.2017.06.013. MR.3761991

[Pos93] L. E. Positsel'skiǔ, Nonhomogeneous quadratic duality and curvature (Russian, with Russian summary), Funktsional. Anal. i Prilozhen. 27 (1993), no. 3, 57-66, 96, DOI 10.1007/BF01087537; English transl., Funct. Anal. Appl. 27 (1993), no. 3, 197-204. MR. 1250981

[Pos11] Leonid Positselski, Two kinds of derived categories, Koszul duality, and comodulecontramodule correspondence, Mem. Amer. Math. Soc. 212 (2011), no. 996, vi+133, DOI 10.1090/S0065-9266-2010-00631-8. MR2830562

[Sch95] W. H. Schikhof, A perfect duality between p-adic Banach spaces and compactoids, Indag. Math. (N.S.) 6 (1995), no. 3, 325-339, DOI 10.1016/0019-3577(95)93200-T. MR.1351151

[Sch11] Peter Schneider, p-adic Lie groups, Grundlehren der Mathematischen Wissenschaften [Fundamental Principles of Mathematical Sciences], vol. 344, Springer, Heidelberg, 2011. MR 2810332

[Sch15] Peter Schneider, Smooth representations and Hecke modules in characteristic $p$, Pacific J. Math. 279 (2015), no. 1-2, 447-464, DOI 10.2140/pjm.2015.279.447. MR3437786

[Ser65] Jean-Pierre Serre, Sur la dimension cohomologique des groupes profinis (French), Topology 3 (1965), 413-420, DOI 10.1016/0040-9383(65)90006-6. MR.180619

[Sor19] C. Sorensen, Hochschild cohomology and p-adic Lie groups, to appear in Münster J. Math.

[Spa88] N. Spaltenstein, Resolutions of unbounded complexes, Compositio Math. 65 (1988), no. 2, 121-154. MR932640

[Sta61] James Dillon Stasheff, Homotopy Associativity of H-spaces, ProQuest LLC, Ann Arbor, MI. Thesis (Ph.D.)-Princeton University, 1961. MR2613327

[Sta18] The Stacks Project Authors, Stacks Project, http://stacks.math.columbia.edu/, retrieved May 2018.

[ST02] P. Schneider and J. Teitelbaum, Banach space representations and Iwasawa theory, Israel J. Math. 127 (2002), 359-380, DOI 10.1007/BF02784538. MR1900706 
[Val14] Bruno Vallette, Algebra + homotopy $=$ operad, Symplectic, Poisson, and noncommutative geometry, Math. Sci. Res. Inst. Publ., vol. 62, Cambridge Univ. Press, New York, 2014, pp. 229-290. MR3380678

[Ven19] Akshay Venkatesh, Derived Hecke algebra and cohomology of arithmetic groups, Forum Math. Pi 7 (2019), e7, DOI 10.1017/fmp.2019.6. MR 4061961

[WE18] C. Wang-Erickson, Deformations of residually reducible Galois representations via $A_{\infty}$ algebra structure on Galois cohomology, preprint, arXiv:1809.02484 2018.

[WE19] C. Wang-Erickson, Higher Yoneda product structures and Iwasawa algebras modulo $p$, preprint, 2019.

[Wit18] S. Witherspoon, An Introduction to Hochschild Cohomology, book draft, retrieved June 2018. Available online.

Department of Mathematics, University of California, San Diego, la Jolla, CaliFORNIA 92093

Email address: csorensen@ucsd.edu 